\title{
Prototype Test Results of the Solar Tower Atmospheric Cherenkov Effect Experiment (STACEE)
}

\author{
M.C. Chantell ${ }^{1}$, D. Bhattacharya ${ }^{2}$, C.E. Covault $^{1}$, M. Dragovan ${ }^{1}$, R. Fernholz ${ }^{3}$, D.T. Gregorich ${ }^{4,5}$,

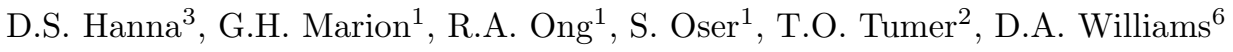 \\ ${ }^{1}$ University of Chicago, Chicago IL 60637, USA \\ ${ }^{2}$ University of California, Riverside, Riverside, CA 92521, USA \\ ${ }^{3}$ McGill University, Montreal, Quebec H3A 2T8, Canada \\ ${ }^{4}$ California State University, Los Angeles, Los Angeles, CA 90032, USA \\ ${ }^{5}$ IPAC, California Institute of Technology, Pasadena, CA 91125, USA \\ ${ }^{6}$ University of California, Santa Cruz, Santa Cruz, CA 95064, USA \\ (Submitted to Nuclear Instruments and Methods in Physics Research A)
}

\begin{abstract}
There are currently no experiments, either satellite or ground-based, that are sensitive to astrophysical $\gamma$-rays in the energy range between 20 and $250 \mathrm{GeV}$. We are developing the Solar Tower Atmospheric Cherenkov Effect Experiment (STACEE) to explore this energy range. STACEE will use heliostat mirrors at a solar research facility to collect Cherenkov light in extensive air showers produced by high energy $\gamma$-rays. Here we report on the results of on-site test work at the solar facility. We demonstrate that the facility is suitable for use as an astrophysical observatory, and using a full scale prototype of part of STACEE, we detect atmospheric Cherenkov radiation at energies lower than any other experiment to date. Based upon these results we are confident that the eventual STACEE instrument will be capable of exploring the $\gamma$-ray sky between 50 and $500 \mathrm{GeV}$ with good sensitivity.
\end{abstract}

PACS codes: 95.55.Ka, 07.85.-m, 42.79.Ek 29.40.Ka 


\section{Introduction}

During the last few years the field of $\gamma$-ray astronomy has been revolutionized by the discovery of over 100 point sources by the EGRET satellite experiment with energies up to $20 \mathrm{GeV}$ [1]. At the same time, improvements in ground-based telescopes using the atmospheric Cherenkov technique have resulted in several recent detections of point sources at energies above $250 \mathrm{GeV}$ [2]. Still, most EGRET sources are not detected above $250 \mathrm{GeV}$. For example, of the large number of Active Galactic Nuclei (AGN) seen by EGRET, only Markarian 421 has been detected by a ground-based instrument [3]. This result implies a spectral cutoff between 20 and $250 \mathrm{GeV}$, and may suggest that high energy $\gamma$-rays are attenuated by photon-photon interactions with the intergalactic infrared background 细, 坷. A measurement of AGN spectral cutoffs as a function of AGN redshift may be used to probe the infrared background, which is sensitive to details of galaxy formation and dark matter composition [6]. As another example, although it is generally believed that supernova remnants (SNR) are important sites for cosmic ray acceleration, to date no clear detection of $\gamma$-rays from SNR has been made by ground-based instruments [7]. There is speculation that the $\gamma$-ray spectra of SNR soften at energies above $20 \mathrm{GeV}$ [8], making it important to observe such objects at as low an energy as possible. For these reasons, the energy range between 20 and $250 \mathrm{GeV}$ is expected to yield a wealth of scientific discovery. To date, however, it remains largely unexplored. The existing satellite experiment (EGRET) has poor sensitivity above $\sim 10 \mathrm{GeV}$ due to its limited collection area, while current ground-based experiments have energy thresholds above $250 \mathrm{GeV}$.

When high energy $\gamma$-rays or cosmic rays enter the Earth's atmosphere, they interact and produce extensive air showers (EAS) of highly relativistic charged particles. These charged particles emit Cherenkov radiation which forms a light pool $\sim 100 \mathrm{~m}$ in radius at ground level. Atmospheric Cherenkov telescopes operate by using large mirrors to collect this light and focus it onto photomultiplier tube cameras. The total amount of Cherenkov light generated by an EAS is directly proportional to the energy of the progenitor and thus, as one goes down in energy, the density of Cherenkov photons on the ground decreases. The energy threshold of this type of instrument is limited by the total mirror collection area. Larger mirror areas yield lower energy thresholds. Currently, the lowest energy threshold obtained by an atmospheric Cherenkov telescope is $\sim 250 \mathrm{GeV}$ for the Whipple Observatory's $10 \mathrm{~m}\left(78.5 \mathrm{~m}^{2}\right.$ mirror area) telescope.

It has been recognized for some time that existing solar power plants represent a potential resource for achieving lower energy thresholds due to their large mirror areas [9, 10]. Over the last few years, we have been exploring the use of heliostat fields at such facilities, with the goal of developing a new experiment called the Solar Tower Atmospheric Cherenkov Effect Experiment (STACEE) [11, 12, 13]. 
The goal of STACEE is to explore the $\gamma$-ray sky in the energy range of $50 \mathrm{GeV}$ to $500 \mathrm{GeV}$. Our current plan is to make use of the National Solar Thermal Test Facility (NSTTF) located in Albuquerque, NM. STACEE will use 48 heliostats, spread out over a $150 \mathrm{~m} \times 300 \mathrm{~m}$ area, to sample a large fraction of the Cherenkov light pool. Each heliostat has a mirror area of $37.2 \mathrm{~m}^{2}$, yielding a total mirror collection area of $\sim 1786 \mathrm{~m}^{2}$.

In 1996, the STACEE collaboration designed and built a prototype secondary telescope and camera for detecting atmospheric Cherenkov showers using the heliostat field of the NSTTF This prototype was installed on the central receiver tower at the NSTTF for a series of tests in August and October 1996. The prototype included electronics and a data readout system for eight photomultiplier tubes viewing eight heliostats.

The purpose of these tests was to explore the feasibility of establishing a $\gamma$-ray observatory at the Sandia site. In particular, our goals for these tests were to establish the following:

- that the environmental conditions at the Sandia site are suitable for doing atmospheric Cherenkov astronomy,

- that the mechanical and optical properties of the heliostats are of sufficient quality for astrophysical observations, and

- that the STACEE concept is viable for a low threshold ground-based $\gamma$-ray detector.

Our results on all three of these topics were excellent. In the remainder of this paper we elaborate our findings.

\section{Detector Concept}

The STACEE detector uses large steerable mirrors, called heliostats, to collect Cherenkov light from extensive air showers. This light is reflected onto a secondary mirror mounted on the central receiver tower. The secondary mirror images the light onto an array of photomultiplier tubes (PMTs) which are mounted on a supporting structure. Because the secondary mirror forms an image of the heliostat field in its focal plane, each PMT may be positioned so that it sees light from only one heliostat. The PMTs together with the supporting structure are referred to as the PMT camera. Figure 2.1 shows a schematic of the STACEE concept. Complete details of the STACEE design concept can be found in [14].

In our 1996 tests the signals from each PMT were capacitively coupled, amplified, and delayed to correct for the varying arrival time of the Cherenkov light at the camera box. PMT signals were combined 
to form a fast trigger. Analog to digital converters (ADCs), time to digital converters (TDCs), and waveform digitizers recorded the pulse amplitudes, times, and waveforms, respectively. The data were read out via CAMAC and GPIB by a 486-based PC. The prototype tests used eight heliostats with eight PMTs. Figure 2.2 shows a schematic of the secondary telescope and camera and Figure 2.3 shows a schematic of the electronics setup. Different sets of heliostats were used in the August and October 1996 tests, as indicated in Figure 2.4.

\section{Results from Environmental Measurements}

The NSTTF is located on the grounds of Kirtland Air Force Base near Albuquerque, New Mexico, at an altitude of $1700 \mathrm{~m}$. Environmental conditions relevant to the STACEE project include:

- the local weather conditions, particularly the expected number of clear nights for astronomical observing,

- the clarity of the atmosphere, particularly the impact of air pollution from nearby Albuquerque that might attenuate Cherenkov light, and

- the ambient light levels at night - including the impact of any extraneous light from Albuquerque.

\subsection{Local Weather Conditions}

Sandia National Laboratories are situated in the southwestern United States in an area with a dry moderate climate. With the exception of seasonal monsoons from July through August, this region receives little precipitation. Atmospheric levels of water vapor, which increases the attenuation of light, are low, and the skies are usually very clear. From meteorological records for Albuquerque (Figure 3.1) we estimate that we will achieve an average of 4.1 hours of cloudless, moonless weather per night during the Sept. through May observing season. We estimate an annual duty cycle of $\sim 10 \%$, which is typical for ground-based atmospheric Cherenkov experiments at good observing sites.

\section{$3.2 \quad$ Atmospheric Clarity}

Atmospheric contaminants, such as aerosols and other pollutants, have the potential to attenuate the Cherenkov light signal as it propagates through the atmosphere above the Sandia site. To determine the clarity of the local atmosphere at Sandia we used stellar photometry to measure the optical transmission of the atmosphere at the site as a function of atmospheric depth and wavelength.

The photometry data were collected over the course of a single night at Sandia at a location approximately 20 meters north of the north edge of the heliostat field. The photometer, as depicted 
schematically in Figure 3.2, consists of a Hamamatsu photon-counting photomultiplier tube (H3460-53) attached to the focus of a Celestron 8" F/10 Schmidt Cassegrain Telescope (SCT). The PMT base housing contains amplifier and discriminator circuitry. Output pulses from the PMT are counted with a scaler which is read out by a laptop PC. The count rate from the PMT is directly proportional to the photon flux falling on its photocathode. The instrument includes a set of standard Johnson UBV photometric filters that allows measurements to be made in the Ultra-violet, Blue, and Visible portions of the spectrum respectively. Figure 3.3 shows the standard UBV photometric response curves. We note that the corrector plate of the SCT is made of crown glass and hence does not transmit below wavelengths of about $340 \mathrm{~nm}$. This cutoff affects measurements in the $\mathrm{U}$ band, and is corrected for in the subsequent analysis of the data.

We made repeated drift scans of four separate bright stars over a five hour period. Drift scans were done for each star using each filter. Each star was scanned at five different zenith angles. The scans included approximately 40 seconds of data before star transit in order to measure the background light level. Figure 3.4 shows the data from a single scan. The background and signal regions are clearly separated, and the boundaries of the signal region are very sharp and well defined.

The total atmospheric transmission factor for each of the UBV wavebands is derived from the observations using standard photometric techniques [15]. The observed flux of star light is found from the data by calculating the average background rate from the data before the the signal peak (see Figure 3.4) and subtracting that value from the average count rate in the signal region of the drift scan, taking care to avoid the regions where the signal is turning on. Error bars shown on subsequent plots are the statistical errors associated with these averages.

To find the unattenuated flux of star light we use Bouguer's law [15] which relates the apparent magnitude of a star $\left(M_{o, \lambda}\right)$ at the zenith as a function of wavelength to $\left(M_{z, \lambda}\right)$ the magnitude as a function of zenith angle $z$ and wavelength:

$$
M_{z, \lambda}=A_{\lambda} \cdot \sec z+M_{o, \lambda}
$$

Note that the logarithm of the photon flux is proportional to the stellar magnitude $M$. To first order, $\sec z$ is proportional to the atmospheric depth (sometimes called the airmass or overburden). From a plot of $M_{z, \lambda}$ versus $\sec z$ the unattenuated stellar magnutide can be found by extrapolating to $\sec z=0$ which corresponds to an airmass of 0 (ie. no atmosphere). Figure 3.5 shows such a plot of the data for the star $\mathrm{Mu}$ Andromeda with the V band filter. 
The atmospheric transmission is calculated as the ratio of the stellar flux on the ground to the incident stellar flux at the top of the atmosphere. A weighted average of results from all four observed stars yields the total atmospheric attenuation for each wavelength band.

Figure 3.6 shows the atmospheric transmission derived from the observations for the blue waveband as a function of zenith angle. The solid line shows the fit of the data to the form:

$$
T=a \exp (-b \sec (z))
$$

where $T$ is the atmospheric transmission factor, $a$ and $b$ are free parameters. Table 1 gives the atmospheric transmission at zenith for all three wavebands.

Figure 3.6 also shows the total transmission predicted from a model of an ideal atmosphere. This model assumes that the only light loss mechanisms are Rayleigh and Mie scattering and absorption by oxygen and naturally-occurring ozone [16. Instrumental effects such as PMT quantum efficiency, wavelength dependent transmission of the crown glass corrector plate, the reflectivity of the mirrors in the telescope, and the appropriate transmission for the UBV filters have all been folded in to the model. We can see that the ideal atmosphere agrees with the observational data to within $3 \%$ (see Table 1). These measurements thereby demonstrate that the clarity of the local atmosphere is not significantly affected by air pollution. Table 1 also shows the predicted clarity for the Whipple Observatory for comparison, calculated assuming an ideal atmosphere; the Sandia and Whipple sites have comparable atmospheric clarity.

\subsection{Ambient Background Light}

In addition to Cherenkov light from extensive air showers, the STACEE detector is also sensitive to ambient light present at the site. This ambient light constitutes the background against which the Cherenkov signal must be detected. One source of ambient background is light from the night sky viewed by the heliostats. This includes air glow, stars in the field-of-view of the heliostats, and backscattered light from artificial sources on the ground. Because the secondary mirror looks down into the heliostat field, a second source of ambient light is light scattering off the ground surrounding the heliostats. This background can be minimized by matching the aperture of each PMT to the size of the heliostat image in the focal plane, so that the PMT sees only the surface of the heliostat and not the ground around it.

Figure 3.7 illustrates the relative contributions of these backgrounds when the heliostats are placed in different orientations. The largest background is seen when the heliostats are pointed to reflect light arriving from zenith into the STACEE telescope. Since we use Winston cones to limit the field of view 
of each PMT to the area of a single heliostat, we expect very little of the reflected light from the ground to be picked up by the PMTs when the heliostats are pointed towards the sky. When the heliostats are turned so that they are "edge on" as seen by the STACEE telescope, the PMTs view only the light reflecting off the ground around the heliostats, and the level of the background drops by a factor of $\sim 1.5$. Since the background light level is reduced by taking the heliostats off the night sky we are confident that the ambient light entering our PMTs is dominated by light from the night sky and not by light produced by nearby Albuquerque.

We calculate the flux of background photons from the zenith by measuring the currents in the PMTs. The current from each PMT due to background can be expressed as:

$$
I=\Phi_{\mathrm{bkg}} e G \Omega \epsilon A,
$$

where $I$ is the PMT current in amperes, $\Phi_{\mathrm{bkg}}$ is the background photon flux, $e$ is the charge of the electron in Coulombs, $G$ is the PMT gain, $\Omega$ is the solid angle in the sky viewed by each heliostat, $\epsilon$ is the efficiency with which a photon incident on the heliostat results in a photoelectron at the PMT, and $A$ is the heliostat collection area. We find that the flux of photons is $4.3 \pm 0.9 \times 10^{12} \mathrm{ph} / \mathrm{m}^{2} / \mathrm{sec} / \mathrm{sr}$. For comparison, the measured flux at a dark mountain site is $2.0 \times 10^{12} \mathrm{ph} / \mathrm{m}^{2} / \mathrm{sec} / \mathrm{sr}$ 17].

\section{Results from Heliostat Performance Tests}

The heliostat field at Sandia is designed to track the Sun and focus its light onto the central receiver tower. An individual heliostat consists of 25 square mirror facets, each $1.22 \mathrm{~m}$ on a side, mounted on a single altitude-azimuth mount. The surface of each facet is deformed slightly using adjustable screws to obtain a parabolic reflective surface. The facets on each heliostat are aimed and focused to produce the smallest possible image of the Sun on the receiver tower.

The elevation and azimuth positions of each heliostat are encoded with a precision of 13 bits over $360^{\circ}$ which corresponds to a precision of $0.04^{\circ}$. Pointing, tracking, and all other aspects of heliostat operations are implemented by a central controller.

To determine the suitability of the existing heliostats at the Sandia site for astronomical measurements, we measured the following:

- pointing accuracy,

- tracking stability, 
- focusing properties of the heliostats, and

- reflectivity.

Details for each of these measurements are described below.

\subsection{Heliostat Pointing Accuracy}

Heliostat pointing accuracy is important to ensure that: 1) all heliostats are viewing the same point in the sky, and 2) the collected light is properly focused onto the secondary optic on the tower.

The pointing accuracy of the heliostats was determined by conducting drift scans of several bright stars. During a single observation, all heliostats were directed to observe a point seven minutes in right ascension ahead of a bright star. The heliostats were halted and the star was allowed to drift through the field of view of the heliostats while the PMT currents were recorded with a scanning ADC. Figure 4.1 shows the light curves obtained for two heliostats during a drift scan of the star Aldebaran. The time of the peak current for each PMT is found from the weighted average of the background-subtracted light curve. Table 2 lists the time, in seconds from the start of the data run, at which the current reached a maximum in each heliostat's light curve.

The data show that the selected heliostats were all aimed at a common point to an accuracy better than $0.08^{\circ}$ with a typical accuracy of $0.04^{\circ}$ which is equal to the resolution of the 13 bit heliostat position encoders. Thus using star transit data we can readily identify heliostats which are not properly aligned and make corrections to fine-tune the heliostat pointing.

The light curves obtained from these drift scans can also be used to determine the field of view for each heliostat. We define the field of view of a heliostat to be the full width half maximum (FWHM) of its recorded light curve for a star transit. From the known angular velocity of Aldebaran, we convert the FWHM of each light curve into a field of view in degrees. For the two light curves shown in Figure 4.1, we obtain an average heliostat field of view of $0.7^{\circ}$.

\subsection{Tracking Stability}

The ability of a heliostat to maintain a celestial object centered in its field of view as the object moves across the sky is referred to as the tracking stability. To examine the stability of the Sandia heliostats, we tracked the bright star Betelgeuse for 18 minutes while recording the PMT currents with scanning ADCs. Figure 4.2 shows the light curves for two of the heliostats. The average current level for each heliostat is stable to within a few percent over the duration of the data run. There is an obvious sawtooth modulation of the currents with a period of $\sim 30$ sec. The sawtooth pattern seen in the lower plot of 
Figure 4.2 results from the heliostat repeatedly being brought on target (the point of maximum current in the PMT) and then slowly drifting off target. The distance through which the target drifts before the heliostat is commanded to update its position is equal to the single bit resolution of the heliostat position encoders. Knowing the apparent motion of Betelgeuse across the sky, we find that the periodicity of the observed sawtooth pattern corresponds precisely to the period expected from the encoder resoultion.

Examination of data from another heliostat shown in the upper plot of Figure 4.2 reveals the same type of sawtooth pattern but with the orientation of the "teeth" in the opposite sense. In this case the heliostat was tracking a position slightly ahead of the target star. Thus the current dropped suddenly when the heliostat moved to reacquire the target and then slowly rose as the star drifted back into the field of view of the heliostat. These results demonstrate how star tracking can be used as a powerful diagnostic tool for evaluating the pointing bias of individual heliostats. We plan to conduct regular star tracking runs as a means of monitoring the heliostat tracking stability. Through the use of these runs and stellar drift scans, we expect to be able to correct the pointing biases of all 48 heliostats to within $0.04^{\circ}$. This accuracy is more than adequate, given STACEE's expected angular resolution of $\sim 0.2^{\circ}$.

\subsection{Optical Properties of the Heliostats}

The critical optical property of the heliostats is their focusing. In order to maximize light collection it is important that the reflected light from a heliostat be focused onto as small an area on the central tower as possible. The minimum acceptable size for the secondary mirrors is determined by the projected heliostat spot size.

The heliostat optics were evaluated by imaging the Sun onto the front face of the tower and recording the images with a CCD camera. Fifteen of the sixteen heliostats used in the two prototype tests were tested in this manner; we were unable to obtain data for one heliostat due to cloudy weather. Figure 4.3 shows a typical heliostat image of the Sun. From these images we have determined the average FWHM heliostat spot size to be less than $2.0 \mathrm{~m}$ diameter. Since the Sun has an angular extent of $0.5^{\circ}$, the size of its image closely matches the expected size of a $50 \mathrm{GeV} \gamma$-ray air shower. Table 3 lists the Sun spot sizes for all fifteen heliostats tested. Figure 4.4 shows the FWHM of the spots as a function of the distance between each heliostat and the target. These data indicate a regular trend towards a tighter concentration of light for heliostats closer to the central tower. We can use this trend to predict the light profile expected at the target from any heliostat in the field. 


\subsection{Heliostat Reflectivity}

To estimate the total light collection efficiency of the STACEE optical system, it is necessary to know the reflectivity of the heliostats as a function of wavelength. The heliostats at Sandia are back-surface silvered glass approximately $3 \mathrm{~mm}$ thick. Because the glass is opaque to UV light, a significant amount of the Cherenkov flux from air showers is unavoidably lost at the heliostat. Measurements performed by Sandia personnel place the average heliostat reflectivity at $80 \%$ for visible light. To measure the reflectivity as a function of wavelength, we designed and constructed a custom reflectometer which was used in the field. The reflectometer uses a collimated light source and a high quality PMT to measure light reflected from the surface of a mirror. The instrument is calibrated using a standard mirror of known reflectivity. Narrow bandpass filters allow the measurement of reflectivity as a function of wavelength. The results are shown in Figure 4.5. These measurements demonstrate that the overall reflectivity is $\sim 85 \%$, and that the UV cutoff occurs near $330 \mathrm{~nm}$. Since the heliostats are already over 20 years old, and have maintained a high degree of reflectivity, we do not expect their optical performance to significantly degrade over time scales relevant to the STACEE project.

\section{$5 \quad$ Prototype Performance Results}

In addition to establishing the suitability of the Sandia site for astronomical observations, we also built and tested a fully functional STACEE prototype (See Figure 2.2). The prototype included a secondary telescope, PMT camera, and data acquisition electronics for 8 channels. In both the August and October tests, we ran the prototype STACEE experiment and investigated the following:

- optical characteristics of the secondary mirrors,

- performance of the analog trigger,

- performance of the digital trigger and coincident trigger rates,

- performance of waveform digitizers.

- energy threshold obtainable, and

The secondary telescope and camera were installed on one of the test bays of the central receiver tower. This bay is $10^{\prime}$ deep, $35^{\prime}$ wide, and $160^{\prime}$ above the heliostat field, and looks out on the field to the north. 


\subsection{Optical performance of the secondary mirrors}

A critical component to the STACEE telescope is the secondary mirror that collects the Cherenkov light from the heliostats and reflects it on to the PMTs. We have explored several design approaches for developing large, highly reflective mirrors at low cost. Two distinct mirror technologies have been tested in the field. During the August test we used a $3 \mathrm{~m}$ diameter multifaceted secondary made with a stretched aluminum membrane mirror technology. During the October test, we used a $1.8 \mathrm{~m}$ diameter back-silvered slumped glass mirror.

\subsubsection{Stretched membrane mirrors}

For the August test we used a secondary mirror with seven facets made of electro-polished stretched aluminum sheet. The individual facets had spherical curvature and were aligned at night by directing a high power searchlight beam onto a heliostat which reflected the light onto the secondary mirror. Each facet produced an optical spot of approximately $4 \mathrm{~cm}$ diameter on a target at the focal plane. To co-align the facets, six facets were covered with black cloth while the seventh facet was adjusted using a three point turnbuckle arrangement. This procedure was repeated until the optical spots from all seven facets were aligned. The aggregate alignment of the secondary system was cross-checked by tracking bright celestial objects (such as the planet Jupiter) and projecting the collected light pattern onto a white lucite board mounted at the focal plane.

\subsubsection{Slumped glass secondary mirror}

For the October test we used a slumped glass parabolic mirror (1.8 m diameter) as the secondary optic. Using a single mirror eliminates the need to align multiple facets. The slumped mirror also provided superior optical surface quality, enough so that the images of the heliostats were clearly visible at the focal plane during daylight. Figure 5.1 shows the images of eight selected heliostats projected onto a white placard at the focal plane. Each of the eight heliostats is cleanly imaged by the secondary, with virtually no optical overlap. The optical spot size for this mirror is less than $1 \mathrm{~cm}$.

\subsubsection{Optical crosstalk}

For an ideal secondary mirror system, all of the reflected light from each heliostat in the field will be focused onto the collecting aperture of a single PMT. However, optical aberrations, imperfections in mirror surface quality, and facet misalignment may combine to defocus the image of the heliostats at the focal plane, causing a small amount of light from one heliostat to enter another heliostat's PMT. We refer to this as optical crosstalk. We measure the amount of crosstalk using Cherenkov light signals 
from cosmic ray air shower data. For each event, time-to-digital converters (TDCs) were used to record the relative arrival times of the Cherenkov light pulses at each PMT. Since the time-of-flight depends upon the unique distance between each heliostat and the secondary, photons that were reflected into the wrong PMT will be out of time with other photons collected from the same Cherenkov event. Analysis of the TDC differences between all combinations of adjacent channels show that the maximum level of crosstalk is approximately $1 \%$. There is no evidence for crosstalk between non-adjacent heliostats. The largest amount of optical crosstalk occurs for physically adjacent channels in the same heliostat row. (Note that with the application of cuts on TDC times and a strategic selection of heliostat locations, optical crosstalk is expected to be negligible for the STACEE experiment).

\subsubsection{Summary of Mirror Tests}

Measurements made with two different mirror technologies show both are suitable for use as the secondary optic. The image sizes are small enough to be contained by $5 "$ diameter Winston cones. Optical crosstalk is small and easily rejected off-line by exploiting the time-of-flight differences between different heliostats.

The single-piece slumped glass secondary gives an optically superior performance over the multifaceted aluminum secondary, and is also easier to mount and align. Therefore this technology is currently considered the most promising for STACEE. The STACEE group continues to develop and improve mirror performance using both technologies.

\subsection{Analog vs. Digital Trigger}

Atmospheric Cherenkov telescopes are typically triggered by requiring a minimum number of PMTs to exceed a preset threshold within a small $(\sim 10 \mathrm{~ns})$ time interval. Two different methods exist for implementing such a trigger. An analog trigger takes the analog signals from each PMT and sums them. This summed signal is sent to a discriminator which produces a trigger if the summed signal exceeds a preset threshold. In a digital trigger each channel is individually discriminated and a trigger is generated when some minimum number of discriminated channels fire within a specified time interval. In deciding on a suitable trigger scheme the following characteristics must be considered:

- sensitivity to accidental triggers resulting from fluctuations in the NSB and afterpulsing in the PMTs,

- sensitivity to local phenomena such as single energetic muons passing close to an individual heliostat, and

- achievable energy threshold. 


\subsubsection{Analog Trigger}

The primary advantage of an analog trigger is that it makes full use of the signal-to-noise improvement that comes from increasing mirror collection area. It has been shown [18] that the achievable energy threshold of an atmospheric Cherenkov telescope goes as:

$$
E_{t h} \propto \sqrt{\frac{\Phi_{b k g} \Omega \tau}{\epsilon A}} .
$$

Here, $\Phi_{b k g}$ is the flux of night sky photons, $\Omega$ is the field of view, $\tau$ is the trigger gate width, $A$ is the mirror collection area, and $\epsilon$ is the efficiency for converting a photon striking a heliostat into a photoelectron in its PMT. Of these quantities, $\Omega$ and $\tau$ are constrained by the physics of air showers and $\epsilon$ is constrained by technology. The most direct way to lower the energy threshold of an atmospheric Cherenkov telescope is to increase the effective mirror area. Since an analog trigger takes full advantage of information contained in the time structure of the PMT pulses, in principle it provides the lowest possible energy threshold.

We tested this form of trigger by pointing all eight heliostats to different parts of the sky, near the zenith, so that their fields of view did not overlap. In this configuration, triggers result only from unwanted sources of background, and not from Cherenkov radiation seen in common by a number of heliostats. We measured the trigger rate as a function of discriminator threshold. The results are shown in Figure 5.2. The data show a clear spectral break near a discriminator threshold of $\sim 200 \mathrm{mV}$. If the camera were triggering only on fluctuations of the background photon flux we would expect to see a single, steeply falling spectrum. To understand the presence of the second spectral component at high thresholds, we use Monte Carlo techniques, together with a model for the optical throughput of the STACEE prototype, to estimate the expected trigger rates resulting from single energetic muons passing near individual heliostats and for single heliostats triggering on small cosmic ray air showers.

To estimate the expected rates, we determine the number of photoelectrons necessary to cause a trigger as a function of discriminator threshold from the known gain of the PMTs $\left(\sim 0.6 \times 10^{6}\right)$, the average PMT pulse width $(\sim 10 \mathrm{~ns}$ FWHM $)$, and the measured losses due to cable attenuation $(\sim 0.3)$. The conversion between photoelectron signal and discriminator threshold is estimated to be 0.28 photoelectrons $/ \mathrm{mV}$. Using the average photon density on the ground, as determined by Monte Carlo, we estimate the energy threshold for protons and single muons as a function of discriminator threshold.

The trigger rate for a single heliostat can then be found from:

$$
R=\Phi A_{\text {eff }} \Omega \epsilon,
$$


where $R$ is the trigger rate, $\Phi$ is the flux of Cherenkov-generating background particles (cosmic ray primaries or muons), $A_{\text {eff }}$ is the effective collection area for a single heliostat, $\Omega$ is the solid angle viewed by a heliostat, and $\epsilon$ is the efficiency for converting photons into photoelectrons.

For cosmic ray air showers we use an effective all-particle cosmic ray flux which has been corrected for the varying Cherenkov yield as a function of composition [12]:

$$
\Phi_{c r}=9.1 \times 10^{-6}\left(\frac{E}{1000 \mathrm{GeV}}\right)^{-1.67} \text { showers } / \mathrm{cm}^{2} / \mathrm{s} / \mathrm{sr},
$$

where $E$ is the effective energy threshold in GeV. For local muons we use the flux of muons above an energy of $7 \mathrm{GeV}$ 19]:

$$
\Phi_{\mu}=1.52 \times 10^{-3} E^{-1.24} \mu / \mathrm{cm}^{2} / \mathrm{s} / \mathrm{sr},
$$

where $E$ is taken to be $7 \mathrm{GeV}$ which is approximately the energy below which Cherenkov production from muons ceases. From drift scan data (see Section 4.1) we find that $\Omega$ for a single heliostat is $\sim 1.2 \times 10^{-4} \mathrm{sr}$. From Monte Carlo simulations of proton showers and individual muons we determine $A_{\text {eff }}$ to be $15400 \mathrm{~m}^{2}$ for protons and $154 \mathrm{~m}^{2}$ for muons. Since all eight heliostats are triggering independently on different parts of the sky the total trigger rate for all eight heliostats combined is eight times the rate for a single heliostat (cosmic ray triggers $+\mu$ triggers). The predicted rates are in good agreement with the measured rates for pulses greater than $200 \mathrm{mV}$, as shown in Figure 5.2. This analysis demonstrates that the simple analog trigger is sensitive to single heliostat triggers resulting from single muons and small cosmic ray showers.

\subsubsection{Digital Trigger}

To test the rate of background triggers for a digital trigger we measured the coincidence rates for different combinations of PMT threshold and multiplicity. As for the analog trigger we performed these measurements with the heliostats pointed at different parts of the sky to determine the rate of unwanted background triggers. Figure 5.3 shows the rate versus threshold curves for three different multiplicity requirements. Note that in contrast to the analog trigger, here we do not see a break in the spectrum, which indicates that the observed rates are due only to fluctuations in the NSB and not a secondary source of unwanted triggers.

\subsubsection{Comparison of Analog and Digital Triggers}

From the background rate versus threshold curves for the two trigger types we see that the analog trigger is sensitive not only to fluctuations of the background photon flux, but also to single heliostat triggers 
from cosmic ray air showers and local muons. The digital trigger appears to be sensitive only to the background photon flux. The analog trigger is sensitive to large amplitude signals in any individual channel whereas the digital trigger demands that the light from an event be spread out over several heliostats. Since we wish to trigger selectively on Cherenkov light from $\gamma$-ray initiated air showers, which will uniformly illuminate heliostats over a $100 \mathrm{~m}$ radius, a digital trigger or a modified analog trigger that incorporates some multiplicity requirement is best suited to our needs.

\subsection{Coincidence Event Studies}

To fully understand the performance of the digital trigger, we studied the rate of coincident events when the heliostats were aimed to a common point in the sky. Using these measurements, we:

- established that the system was triggering on cosmic ray showers,

- investigated the zenith angle dependence,

- investigated the rates as a function of heliostat canting angle, and

- determined the energy threshold for the prototype telescope.

\subsubsection{Cosmic Ray Triggers}

To establish that the STACEE prototype is triggering on genuine air shower events we took coincident data with the heliostats in two separate viewing configurations: all heliostats viewing separate areas of the sky near the zenith (random mode), and all heliostats simultaneously viewing the zenith (coincident mode). A digital trigger condition of any four out of eight (4/8) PMTs at a PMT threshold of $31 \mathrm{mV}$ was used, and PMT pulse height and timing information were recorded. When the heliostats were in random mode we expected no coincidences due to cosmic-ray air showers. In this configuration we observed an event rate of $0.2 \mathrm{~Hz}$. When the heliostats were placed in coincident mode the event rate increased to 5.1 Hz. This indicates that our rate of genuine coincident events due to cosmic ray air showers was $4.9 \mathrm{~Hz}$.

To further establish that the STACEE prototype was triggering on cosmic ray air showers we examine the PMT pulse height distribution from the data taken with the heliostats viewing the zenith in coincident mode. Since the field of view of each heliostat is well matched to the angular extent of an air shower, the total light collected by a channel is directly related to the energy of the air shower progenitor. We expect that the spectrum of observed pulse heights in the individual channels should match the differential cosmic ray energy spectrum at $100 \mathrm{GeV}$. In this energy range, the cosmic ray spectrum has a power-law dependence on energy, $d N / d E \propto E^{\alpha}$, where $\alpha$ is -2.65 . We analyzed 35 minutes of data taken 
with the heliostats observing the zenith in coincidence mode. The digitized pulse heights of all channels exceeding threshold were combined to build a pulse height spectrum (Figure 5.4). The rising part of the observed spectrum is due to a convolution of trigger inefficiencies for small pulses and fluctuations in shower brightness at energies near threshold. The trigger becomes fully efficient for events with pulse heights above 60 digital counts. A fit to the falling edge of the spectrum yields a power law index $\alpha$ of $-2.7 \pm 0.1$ (statistical error only), which agrees well with the known differential index of the cosmic ray spectrum in this energy range. Thus, we are confident that we were in fact triggering on Cherenkov light from cosmic ray air showers.

\subsubsection{Zenith angle effects on system trigger}

Because of the celestial motion of sources across the sky, $\gamma$-ray observations must be conducted over a range of zenith angles. The effective energy threshold of an atmospheric Cherenkov telescope varies with zenith angle due to the increased atmospheric overburden. As the overburden increases air showers develop further away from the ground, resulting in: (1) an increase in the attenuation of the Cherenkov light, and (2) a spread in the Cherenkov light pool over a larger area, reducing the photon density on the ground. Both of these effects are proportional to the increase in atmospheric depth with zenith angle and therefore, we expect the event rate to decrease with zenith angle. Figure 5.5 shows the observed

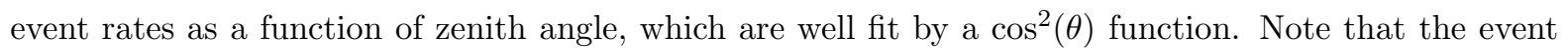
rates fall off slowly out to a zenith angle of $30^{\circ}$, after which they fall more steeply. In order to maximize sensitivity, STACEE will concentrate on observing sources within $30^{\circ}$ of the zenith.

\subsubsection{Effect of heliostat canting on event rates}

Since the heliostats have small fields of view, maximum trigger sensitivity is obtained when the heliostats are pointed to the shower interaction region (approximately $10 \mathrm{~km}$ above sea level). The interaction region is defined as the location where most of the Cherenkov light is generated for events that land on the geometric center of the heliostat field. Tracking the heliostats to observe the interaction region, rather than towards the source at infinity, requires a small adjustment in the pointing angle of each individual heliostat. This adjustment is called "canting" because each heliostat is canted slightly inwards so as to observe the interaction region.

Figure 5.6 illustrates the application of correct heliostat canting. Figure 5.6a shows the fields of view of the heliostats in the case where all heliostats are tracking parallel in the direction of the source at infinity. In this case, coverage of the event track is incomplete. Figure $5.6 \mathrm{~b}$ shows the heliostats correctly canted so that each heliostat will collect light from the full longitudinal development of the air shower. 
The interaction region corresponds to the point of shower maximum in the development of an air shower and occurs at an atmospheric depth of $270 \mathrm{~g} / \mathrm{cm}^{2}(\sim 10 \mathrm{~km}$ altitude) for showers initiated by $50 \mathrm{GeV}$ $\gamma$-rays. Figure 5.7 shows the effect of changing the heliostat canting angle on the trigger rate. The distribution is well fit by a Gaussian with a mean of $0.15^{\circ}$, corresponding to a depth of shower maximum consistent with the expected value for cosmic rays at energies of a few hundred $\mathrm{GeV}$ which compose the bulk of the event triggers (see section 5.3.5).

\subsubsection{Performance of Waveform Digitizers}

A key goal of the on-site tests was to investigate the suitability of waveform digitizers for pulse timing and amplitude measurement. As part of the electronics setup, we used Tektronix 644A and Tektronix 740A digital oscilloscopes to digitize the PMT waveforms for all eight phototubes. The Tektronix 644A scope had a sampling rate of 2 GSample/sec, while the 740A had a sampling rate of 500 MSample/sec. Sampling at 1 GSample/s was simulated with the $644 \mathrm{~A}$ by averaging consecutive data points. Each scope had a dynamic range of 8 bits. The digitized waveforms were read out over a GPIB interface and saved to disk. The GPIB interface readout introduced a significant deadtime (nearly 0.4 sec per event), and thus the waveform digitizers were only read out on selected runs.

For vertical showers, the maximum difference in shower arrival times at two adjacent heliostats spaced $10 \mathrm{~m}$ apart is about $0.6 \mathrm{~ns}$. (Note that here we refer only to shower arrival times at the heliostats in the field and not the difference in shower arrival times at the secondary telescope on the tower). Thus, the spread in photon arrival times is smaller than the expected measurement precision of $\sim 1$ ns. Hence, measurements of Cherenkov showers taken at zenith serve as a calibration beam for timing measurements, and the spread in the difference of arrival times between two adjacent heliostats is dominated by the timing resolution of the electronics.

The expected location of the Cherenkov pulse in each digitized waveform can be determined from the known detector geometries and cable delays. Using data within a $15 \mathrm{~ns}$ window centered around the expected pulse location, the arrival time of the Cherenkov pulse was defined to be the centroid of all data points exceeding a threshold of $30 \mathrm{mV}$. We find that this technique yields better fitted times with a smaller spread than other methods, including a Gaussian fit to the pulse shape.

To determine the timing resolution, we calculated the quantity:

$$
\Delta t=t_{2}-\frac{t_{1}+t_{3}}{2},
$$

where, $t_{1}, t_{2}$, and $t_{3}$ are the measured pulse arrival times at three consecutive heliostats in the same row. 
We calculate the RMS spread in $\Delta t, \sigma(\Delta t)$, for timing measurements made with the waveform digitizers and for measurements made using conventional TDCs on the same data. Assuming independent measurements, the timing resolution, $\sigma_{t}$, is equal to:

$$
\sigma_{t}=\frac{\sigma(\Delta t)}{\sqrt{\frac{3}{2}}} .
$$

We find that the waveform digitizers give a narrower spread in $\Delta t$ than the TDCs for all pulseheights (See Table 4). In addition, the digitizers allow us to fit pulse arrival times for many pulses which were too small to trigger the TDC. Thus, using the waveform digitizers, we are able to reconstruct arrival times for events which cannot be reconstructed by TDCs alone.

We also studied the applicability of waveform digitizers for pulse charge measurement. By summing the digitized voltages recorded within an integration region around the pulse arrival time, we determined the total charge in the pulse. We find that the waveform digitizers give charge measurements which agree with those found from conventional ADCs.

\subsubsection{Energy Threshold}

We estimate the effective energy threshold for cosmic rays and $\gamma$-rays of the eight heliostat configuration used during the October test. We calculate the energy threshold for cosmic rays from the measured event rate while observing the zenith. The observed rate is related to the energy threshold by:

$$
R=\Phi_{c r} A_{\mathrm{eff}} \Omega
$$

where $R$ is the observed rate, $\Phi_{c r}$ is the flux of cosmic ray showers, $A_{\text {eff }}$ is the effective collection area, and $\Omega$ is the solid angle acceptance of the instrument. For the flux of cosmic rays we use the equivalent proton flux given by Equation 6 .

The maximum observed trigger rate was $5.1 \mathrm{~Hz}$, using a digital trigger with a multiplicity of $4 / 8$ PMTs and a tube threshold of $31 \mathrm{mV}$. The accidental trigger rate in this configuration was $0.2 \mathrm{~Hz}$, and was subtracted from the total rate to yield $4.9 \mathrm{~Hz}$.

The effective collection area and solid angle acceptance for proton-induced air showers are calculated from Monte Carlo simulations. We simulate proton air showers using MOCCA [21, a well-established air shower Monte Carlo. The simulated showers are then processed through a detailed ray tracing simulation of the Sandia heliostat field. This simulation takes into account the measured focusing properties of the heliostats and secondary mirror, reflectivity losses, and ambient background light levels. Proton showers 
were simulated at energies from $50 \mathrm{GeV}$ to $2 \mathrm{TeV}$ and weighted according to the cosmic ray spectrum. The simulated showers were then dropped randomly onto the heliostat field over a $100 \mathrm{~m}$ radius area around the heliostats, and the incident angle of the showers were randomly varied within $1.0^{\circ}$ of zenith. The effective collection area calculated this way is:

$$
A_{\mathrm{eff}}=1.9 \pm 0.7 \times 10^{8} \mathrm{~cm}^{2}
$$

while the solid angle acceptance is:

$$
\Omega=3.7 \pm 0.2 \times 10^{-4} \mathrm{sr}
$$

Note that the solid angle acceptance of the eight heliostats in coincidence is larger than the individual heliostat acceptances. This is due to the fact that the system is triggering on air showers which have an angular extent of $0.5^{\circ}$.

Applying Equation 8 together with Equation 6 yields a cosmic ray energy threshold of:

$$
E_{\mathrm{cr}}=295_{-50}^{+96} \mathrm{GeV}
$$

An alternative way of estimating the effective energy threshold is to compare the measured photoelectron yields to the expected photon densities on the ground as a function of primary energy. We use the MOCCA simulation to determine the average photon density on the ground within $100 \mathrm{~m}$ of the shower core, as a function of energy (see Figure 5.8).

Figure 5.4 shows the pulse height spectrum built up from all PMT pulses which exceeded trigger threshold for observations made at zenith. We define the trigger threshold pulse height as the pulse height above which the integrated area of the power law fit equals the area under the data curve (ie. the trigger rate predicted by the fit equals the observed rate). This corresponds to the minimum pulse height needed to trigger a PMT. The trigger pulse height is found to be:

$$
P_{\text {trig }}=33_{-6}^{+8} \text { digital counts }
$$

where the uncertainty is due to the uncertainty in the spectral index of the fit. Knowing the rate at which charge is converted to digital counts in the $\operatorname{ADC}(Q=0.25 \mathrm{pC} /$ digital count $)$, the PMT gain $\left(G_{\mathrm{pmt}}=6.3 \pm 1.3 \times 10^{5}\right)$ and the gain of the pre-amp $\left(G_{\mathrm{amp}}=\times 10\right)$ we can relate the equivalent number of photoelectrons $\left(N_{p e}\right)$ at trigger threshold to the trigger threshold pulse height by: 


$$
P_{\text {trig }}=\frac{N_{p e} G_{\mathrm{pmt}} G_{\mathrm{amp}} e}{Q},
$$

where $e$ is the charge of an electron. From this we find:

$$
N_{p e}=8.3_{-1.5}^{+2.0} \text { photoelectrons. }
$$

To convert from photoelectrons at the photocathode to photons striking the heliostat $\left(N_{\text {phot }}\right)$ we divide by the average PMT quantum efficiency (0.21, a convolution of the Cherenkov spectrum on the ground and the wavelength-dependent quantum efficiency of the PMT), and by the collection efficiencies due to heliostat reflectivity (0.8), secondary mirror reflectivity (0.85), heliostat focusing efficiency on the secondary (0.8), secondary focusing efficiency on the PMT winston cone (0.85), and the throughput of the Winston cone (0.9). This gives the number of photons incident on a heliostat:

$$
N_{\text {phot }}=95_{-23}^{+27} \text { photons, }
$$

where a $15 \%$ systematic error has been included to account for uncertainties in the efficencies and reflectivities.

Dividing by the projected area of a heliostat $\left(29 \mathrm{~m}^{2}\right)$ gives the photon density $(\rho)$ on the ground necessary to trigger a heliostat channel:

$$
\rho=3.3_{-0.8}^{+0.9} \text { photons } / \mathrm{m}^{2} .
$$

From Figure 5.8 we can use $\rho$ to estimate the lowest energy $\gamma$-ray that will produce a high enough photon density to trigger the experiment. We find the energy threshold, for $\gamma$-rays from a source at the zenith, to be:

$$
E_{\gamma}=74_{-14}^{+17} \mathrm{GeV}
$$

For cosmic rays, the same photon density corresponds to an energy threshold of:

$$
E_{\text {cr }}=385_{-60}^{+55} \mathrm{GeV} .
$$

This energy threshold agrees within error with that obtained from the rate calculation. 


\section{Summary}

We are developing a novel atmospheric Cherenkov experiment called STACEE that will use solar heliostat mirrors at the National Solar Thermal Test Facility (NSTTF). Two on-site tests have been conducted. These tests have demonstrated that the heliostat field at the NSTTF is suitable for use as the primary optical component of an astrophysical $\gamma$-ray detector. Weather and atmospheric conditions at the site compare favorably to conditions at existing observatories. Measurements of the ambient background light levels show that the close proximity of the site to Albuquerque will not adversely effect the performance of STACEE. The mechanical and optical performance of the heliostat field is found to be excellent, in most cases greatly exceeding our requirements.

The NSTTF is a scientific research facility, and so possesses an excellent support infrastructure. This infrastructure includes heavy lift capability, a high bay, a complete machine shop, and other facilities necessary for developing and running an experiment such as STACEE. We feel that the NSTTF site is well suited in all respects for astrophysical observations.

The STACEE prototype's secondary telescope, camera, and associated electronics also performed to expectations. The secondary optics produce well-focused heliostat images that are completely contained by the PMT Winston cones. Measurements of cosmic ray air showers show that the system is stable and responds as expected. The measurements indicate that our prototype detector had a $\gamma$-ray energy threshold below $100 \mathrm{GeV}$, and that the STACEE concept can successfully obtain lower energy thresholds than existing ground-based techniques.

\section{Acknowledgments}

We are grateful to the Physics Division of Los Alamos National Laboratory for loans of electronics equipment. We thank the staff at the NSTTF and in particular, acknowledge the contributions made by J.M. Chavez, R.M. Edgar, C.M. Ghanbari, D. Johnson, J.J. Kelton, L. Killian, and R. Tucker. We also acknowledge the assistance of E. Pod, the engineering staff of the Yerkes Observatory, the personnel of the McGill Physics Department shop. We wish to thank M. Cresti for the use of a $1.8 \mathrm{~m}$ mirror, and J. Carlstrom of the University of Chicago for the loan of a digital oscilloscope. This work was supported by the National Science Foundation, the Institute of Particle Physics of Canada, the Natural Sciences and Engineering Research Council, and the California Space Institute. TOT wishes to acknowledge the support of the University of California, Riverside, Vice Chancellor of Research and College of Natural and Agricultural Sciences. CEC wishes to acknowledge support from the Louis Block Fund of the University of Chicago. RAO wishes to acknowledge the support of the Grainger Foundation and the 
Physical Sciences Division and the Enrico Fermi Institute of the University of Chicago. 


\section{References}

[1] D. Thompson et al., Astrophys. J. Supp. 101, 259 (1995).

[2] R.C. Lamb et al., Proc. of the 1994 Snowmass Summer Study, Particle and Nuclear Astrophysics and Cosmology in the Next Millennium, ed. E.W. Kolb and R.D. Peccei (World Scientific, Singapore) 295 (1995).

[3] M. Punch et al., Nature 358, 477 (1992).

[4] F.W. Stecker and O.C. De Jager, Astrophys. J. 415, L71 (1993).

[5] S.D. Biller et al, Astrophys. J. 445, 227 (1995).

[6] D. MacMinn and J.R. Primack, Space Sci. Rev. 75, 413 (1996).

[7] R.W. Lesard et al., Proc. 24th Int. Cosmic Ray Conf. (Rome) 2, 475 (1995).

[8] L. O'c. Drury, F.A. Aharonian, and H.J. Volk, Astron. Astrophys. 287, 959 (1994).

[9] S. Danaher et al., Solar Energy 28, 335 (1982).

[10] O.T. Tumer et al., Nucl. Phys. B (Proc. Suppl.) 14A, 351 (1990).

[11] O.T. Tumer et al., Proc. 22nd Int. Cosmic Ray Conf. (Dublin) 2, 635 (1991).

[12] R.A. Ong et al., Astroparticle Phys. 5, 353 (1996).

[13] R.A. Ong et al., Proc. Towards a Major Atmospheric Detector-IV (Padova, Italy), ed. M. Cresti, $261(1995)$

[14] The STACEE Collaboration, The Solar Tower Atmospheric Cherenkov Effect Experiment (STACEE) Design Report, EFI Preprint 97-17, March 1997 (unpublished).

[15] C.R. Kitchin, Astrophysical Techniques (Adam Hilger, London), p. 304 (1991).

[16] M.C. Chantell, UV Imaging of Extensive Air Showers at TeV Energies, Ph.D. Dissertation, University of Arizona, 1995 (unpublished).

[17] R. Mirzoyan and E. Lorenz, Measurement of the Night Sky Light Background at La Palma, MPI-PhE/94-35, (1994).

[18] T.C. Weekes, Phys. Reports 160, 1 (1988). 
[19] O.C. Alkofer and P.K.F. Grieder, Physics Data - Cosmic Rays on Earth (Fach-InformationsZentrum, Karlsruhe) (1984).

[20] C.W. Allen, Astrophysical Quantities, 3rd Edition (The Athlone Press, London), p. 201 (1991).

[21] A.M. Hillas, Proc. 19th Int. Cosmic Ray Conf. (La Jolla) 3, 445 (1985). 


\begin{tabular}{|c|c|c|c|}
\hline Waveband & $\mathrm{U}$ & $\mathrm{B}$ & $\mathrm{V}$ \\
\hline Measured (Sandia) & 0.548 & 0.707 & 0.813 \\
\hline Model Atmosphere (alt. $=1700 \mathrm{~m})$ & 0.562 & 0.726 & 0.841 \\
\hline Predicted Whipple (alt. $=2300 \mathrm{~m})$ & 0.590 & 0.760 & 0.861 \\
\hline
\end{tabular}

Table 1: Atmospheric transmission factors for star light from the zenith. Note that $1700 \mathrm{~m}$ corresponds to the altitude of Sandia. 


\begin{tabular}{|c|c|c|}
\hline Heliostat ID & Transit Time (s) & $\Delta^{\circ}$ \\
\hline $8 \mathrm{E} 3$ & 433 & 0.05 \\
$8 \mathrm{E} 4$ & 433 & 0.05 \\
$8 \mathrm{E} 5$ & 402 & -0.08 \\
$8 \mathrm{E} 6$ & 418 & -0.01 \\
$10 \mathrm{E} 4$ & 437 & 0.07 \\
$10 \mathrm{E} 5$ & 421 & 0.00 \\
$10 \mathrm{E} 6$ & 412 & -0.04 \\
$10 \mathrm{E} 7$ & 413 & -0.03 \\
\hline
\end{tabular}

Table 2: Star transit times for each heliostat for the drift scan of Aldebaran. The angular separation $\left(\Delta^{\circ}\right)$ of each heliostat, relative to the average of the eight transit times, is shown. 


\begin{tabular}{|c|c|c|c|c|c|c|}
\hline \multirow{2}{*}{$\begin{array}{l}\text { Helio } \\
\text { ID }\end{array}$} & \multirow{2}{*}{$\begin{array}{l}\text { Distance } \\
\text { to target } \\
\qquad(\mathrm{m})\end{array}$} & \multirow{2}{*}{$\begin{array}{l}\text { FWHM } \\
\text { (m) }\end{array}$} & \multicolumn{3}{|c|}{$\begin{array}{l}\text { Radius (m) } \\
\text { to contain luminance }\end{array}$} & \multirow{2}{*}{$\begin{array}{l}\text { Luminance within } \\
2.0 \text { m diameter } \\
\text { (percent) }\end{array}$} \\
\hline & & & $50 \%$ & $80 \%$ & $90 \%$ & \\
\hline 8E3 & 103.9 & 0.93 & 0.40 & 0.63 & 0.82 & 94 \\
\hline $8 \mathrm{E} 4$ & 106.9 & 0.94 & 0.41 & 0.64 & 0.83 & 94 \\
\hline $8 \mathrm{E} 5$ & 110.4 & 1.27 & 0.61 & 0.97 & 1.25 & 82 \\
\hline 8E6 & 114.6 & 0.99 & 0.44 & 0.68 & 0.85 & 94 \\
\hline $10 \mathrm{E} 4$ & 131.4 & 1.04 & 0.45 & 0.70 & 0.87 & 94 \\
\hline $10 \mathrm{E} 5$ & 134.3 & 1.10 & 0.51 & 0.80 & 0.99 & 90 \\
\hline $10 \mathrm{E} 6$ & 137.8 & 1.08 & 0.50 & 0.79 & 0.99 & 90 \\
\hline $10 \mathrm{E} 7$ & 141.9 & 1.14 & 0.53 & 0.84 & 1.05 & 88 \\
\hline $12 \mathrm{~W} 1$ & 158.8 & 1.21 & 0.57 & 0.88 & 1.10 & 86 \\
\hline $12 \mathrm{E} 1$ & 158.8 & 1.29 & 0.63 & 1.01 & 1.33 & 80 \\
\hline $12 \mathrm{E} 2$ & 159.4 & 1.24 & 0.61 & 1.04 & 1.50 & 78 \\
\hline $14 \mathrm{E} 1$ & 198.2 & 1.67 & 0.81 & 1.25 & 1.53 & 65 \\
\hline $14 \mathrm{E} 2$ & 198.6 & 1.56 & 0.70 & 1.06 & 1.29 & 76 \\
\hline 14E3 & 199.6 & 1.53 & 0.69 & 1.06 & 1.29 & 77 \\
\hline $14 \mathrm{E} 4$ & 201.0 & 1.63 & 0.77 & 1.18 & 1.45 & 69 \\
\hline
\end{tabular}

Table 3: Heliostat Sun spot sizes as measured from background subtracted CCD images taken at Sandia. 


\begin{tabular}{|lccc|}
\hline Timing Method & Small Pulseheights & Large Pulseheights & All Data \\
& $\sigma_{t}$ & $\sigma_{t}$ & $\sigma_{t}$ \\
\hline \hline Conventional TDCs & $0.82 \mathrm{~ns}$ & $0.87 \mathrm{~ns}$ & $0.86 \mathrm{~ns}$ \\
Waveform Digitizers & $0.73 \mathrm{~ns}$ & $0.51 \mathrm{~ns}$ & $0.60 \mathrm{~ns}$ \\
\hline
\end{tabular}

Table 4: Comparison of measured timing resolution for conventional TDCs and 1 GSample/sec waveform digitizers. The waveform digitizers have superior timing resolution at all pulseheights. 


\section{STACEE CONCEPT}

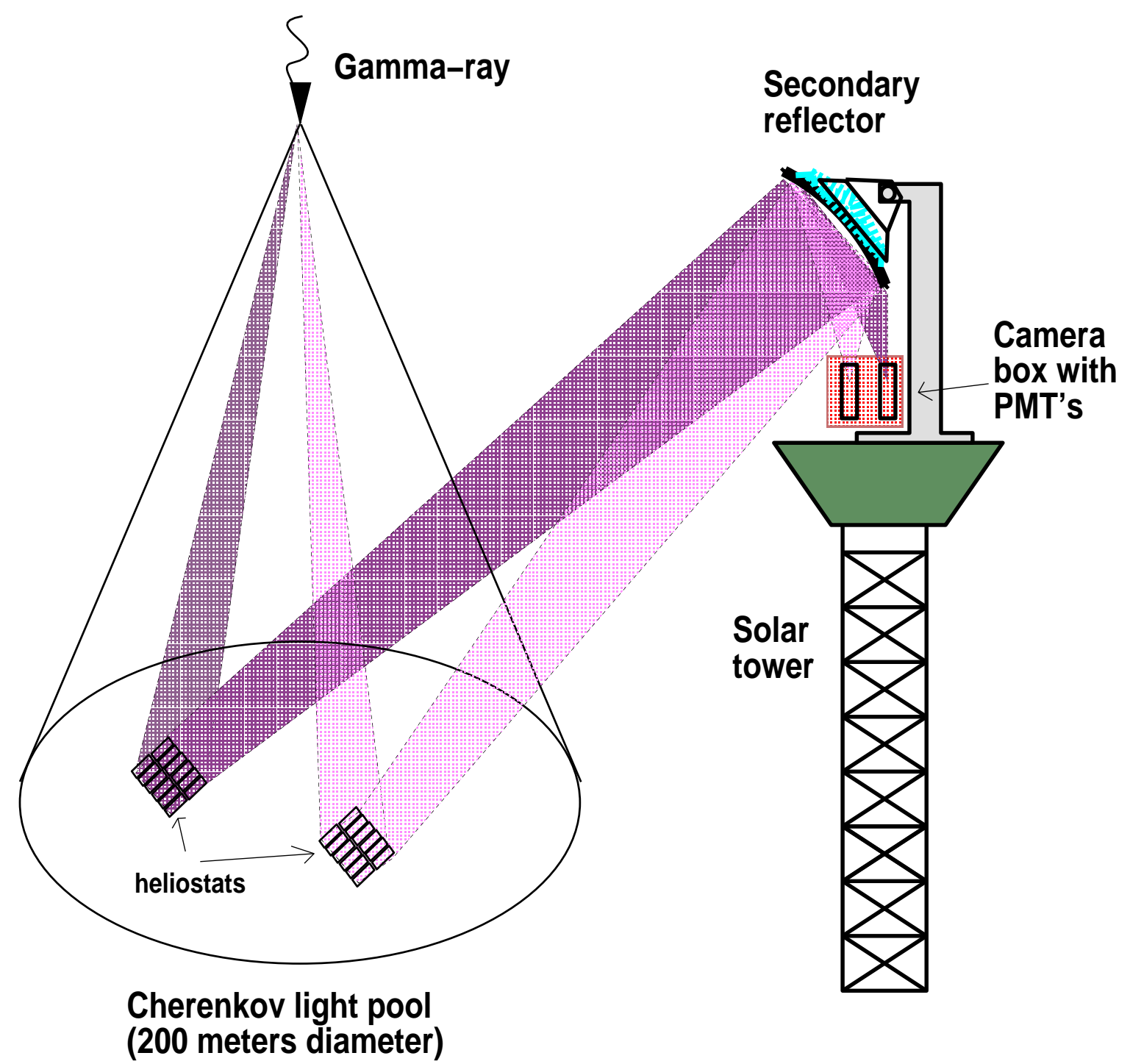

Figure 2.1: The STACEE concept. Cherenkov light produced in the air shower created by an astrophysical $\gamma$-ray is beamed to the ground. Solar heliostat mirrors reflect this light to a secondary collector on the central tower which in turn reflects it to a camera of photomultiplier tubes. 


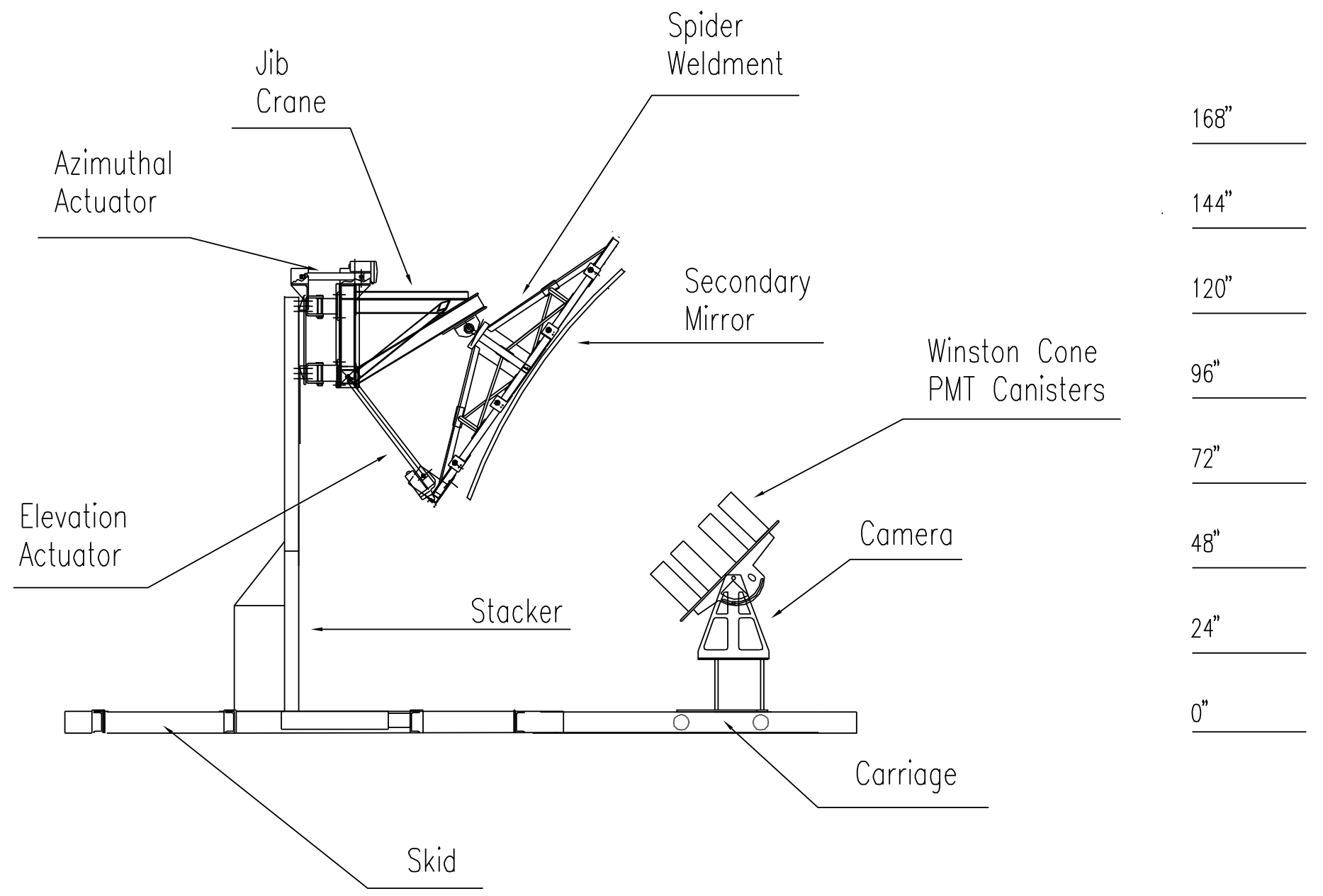

Figure 2.2: STACEE telescope design, including secondary optic and camera. The STACEE instrument will consist of three such telescope modules. One of these has already been constructed and tested successfully in the field. Dimensions are given by the scale on the right. 


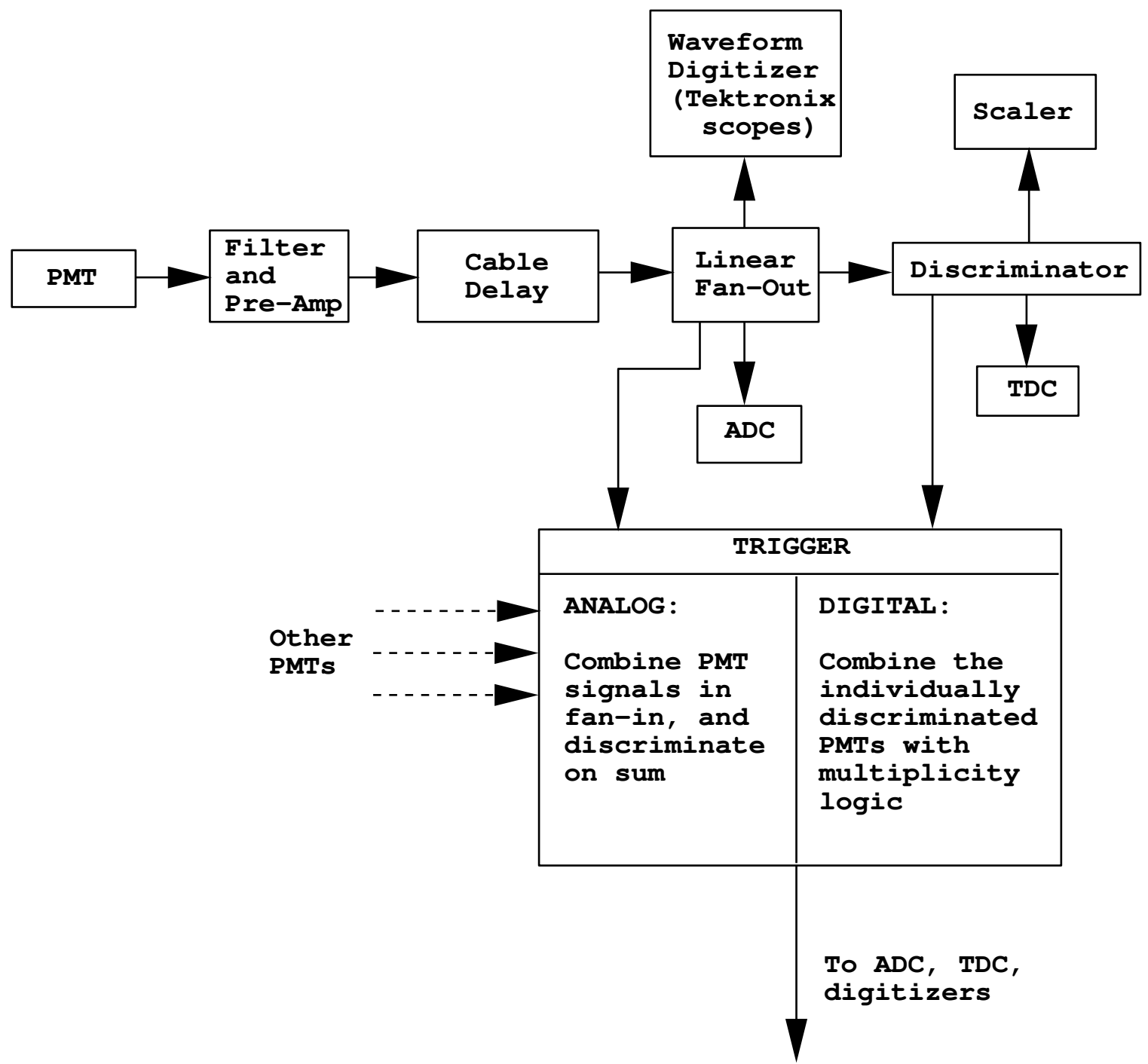

Figure 2.3: Schematic of the electronics setup used for the prototype tests (one channel of eight). Each PMT signal was AC-coupled and amplified. Cable delays compensated for the varying times-of-flight between heliostats and the central tower. Each signal was then split by a fan-out. Analog copies of the signal went to waveform digitizers and ADC units. The signal was also discriminated, and the discriminated outputs drove scaler units and stoped TDCs. The trigger was formed by combining signals from all eight PMTs, and the trigger gated the waveform digitizers and ADCs, and started the TDC units. 


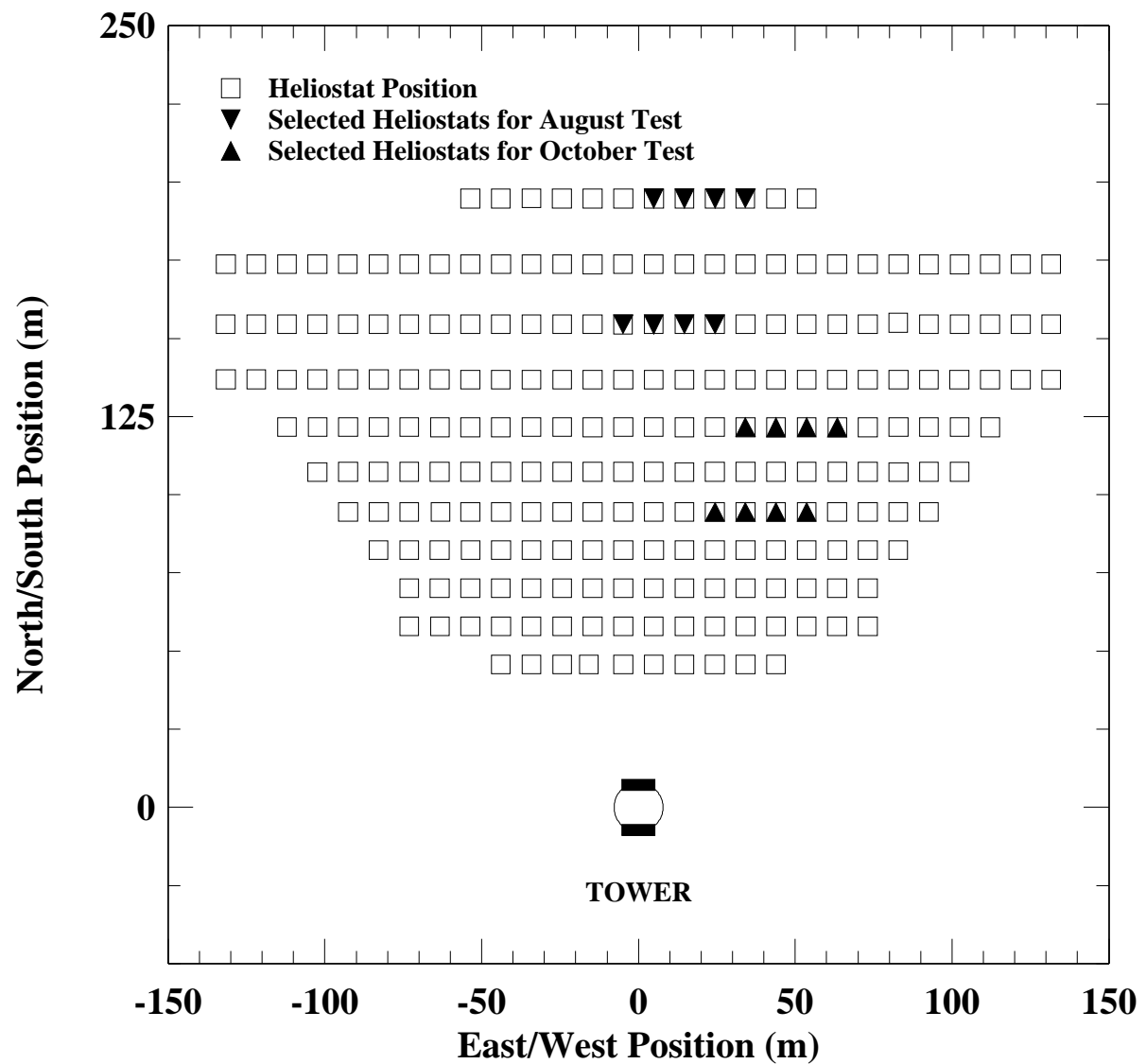

Figure 2.4: Layout of the Sandia heliostat field. Each square represents the location of a single heliostat. The sizes of the squares are not proportional to the physical size of the heliostats. The two different eight-heliostat configurations used in the August and October tests are shown. 


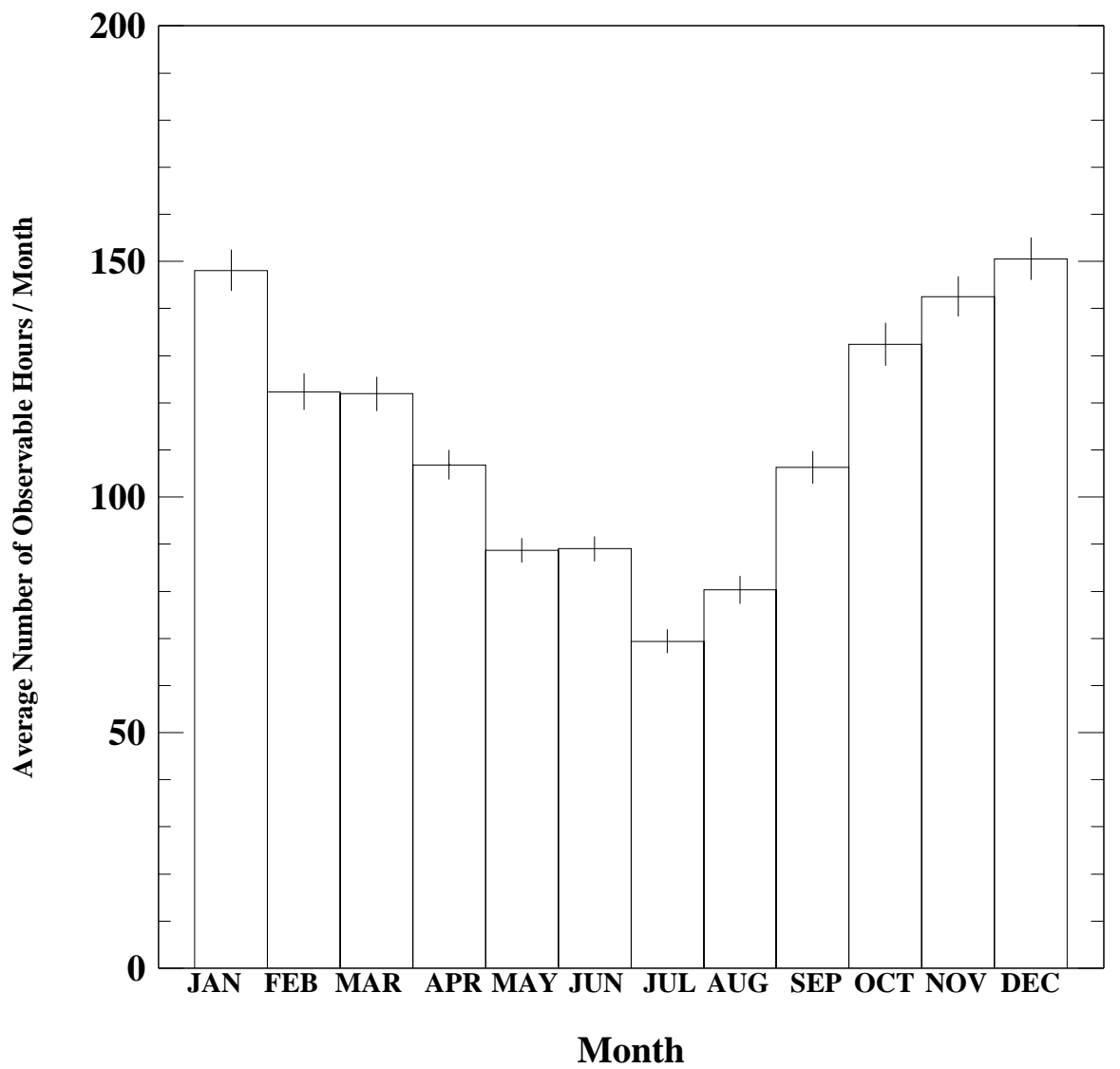

Figure 3.1: Average number of hours of clear, moonless observing time expected per month. The expected fraction of clear skies is taken from daily meteorological records for rain fall and percent sunshine for Albuquerque from 1948 to the present. 
Celestron 8" SCT $\mathrm{f} / 10$

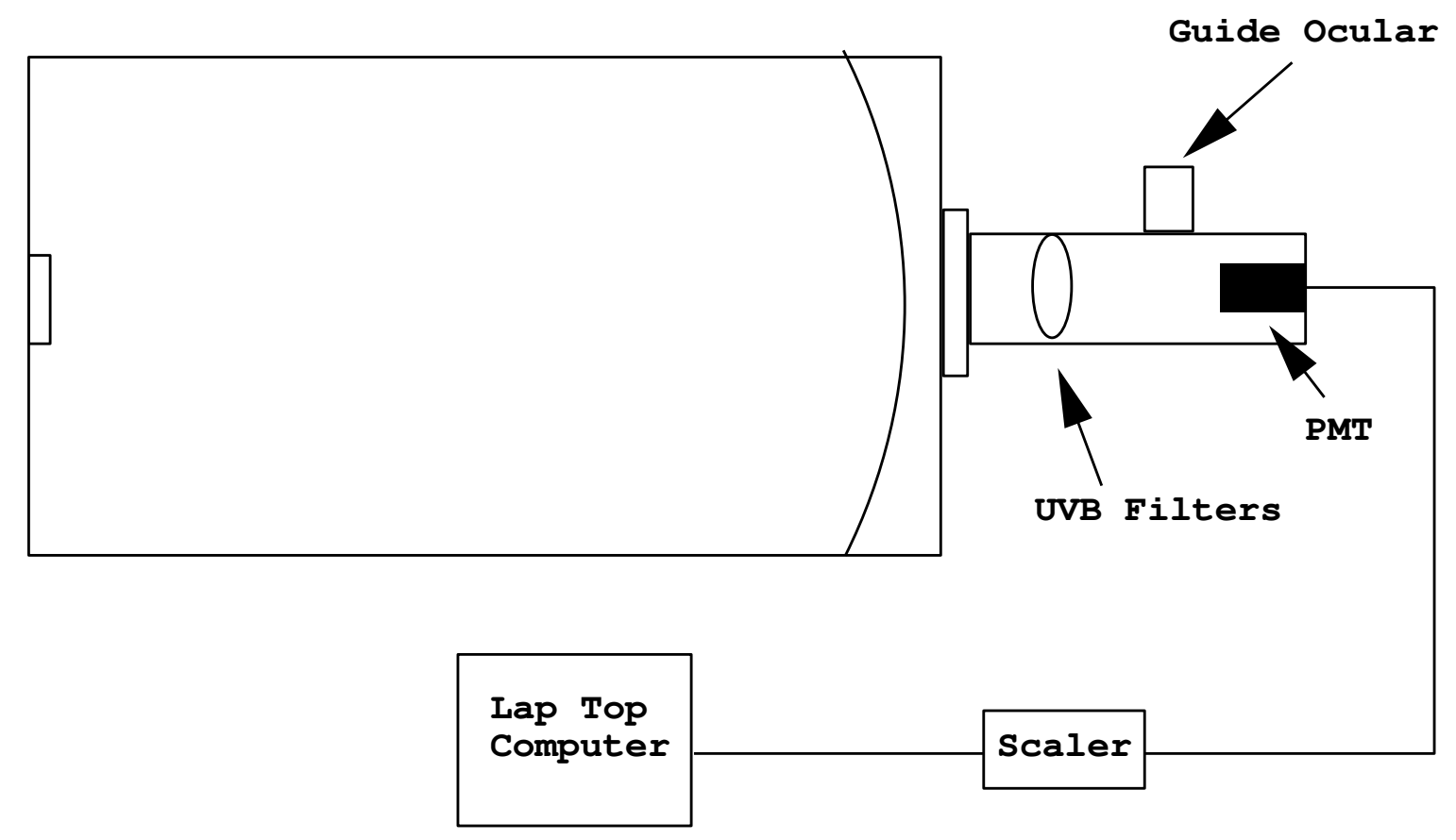

Figure 3.2: Schematic drawing of the photometer setup used at Sandia. 


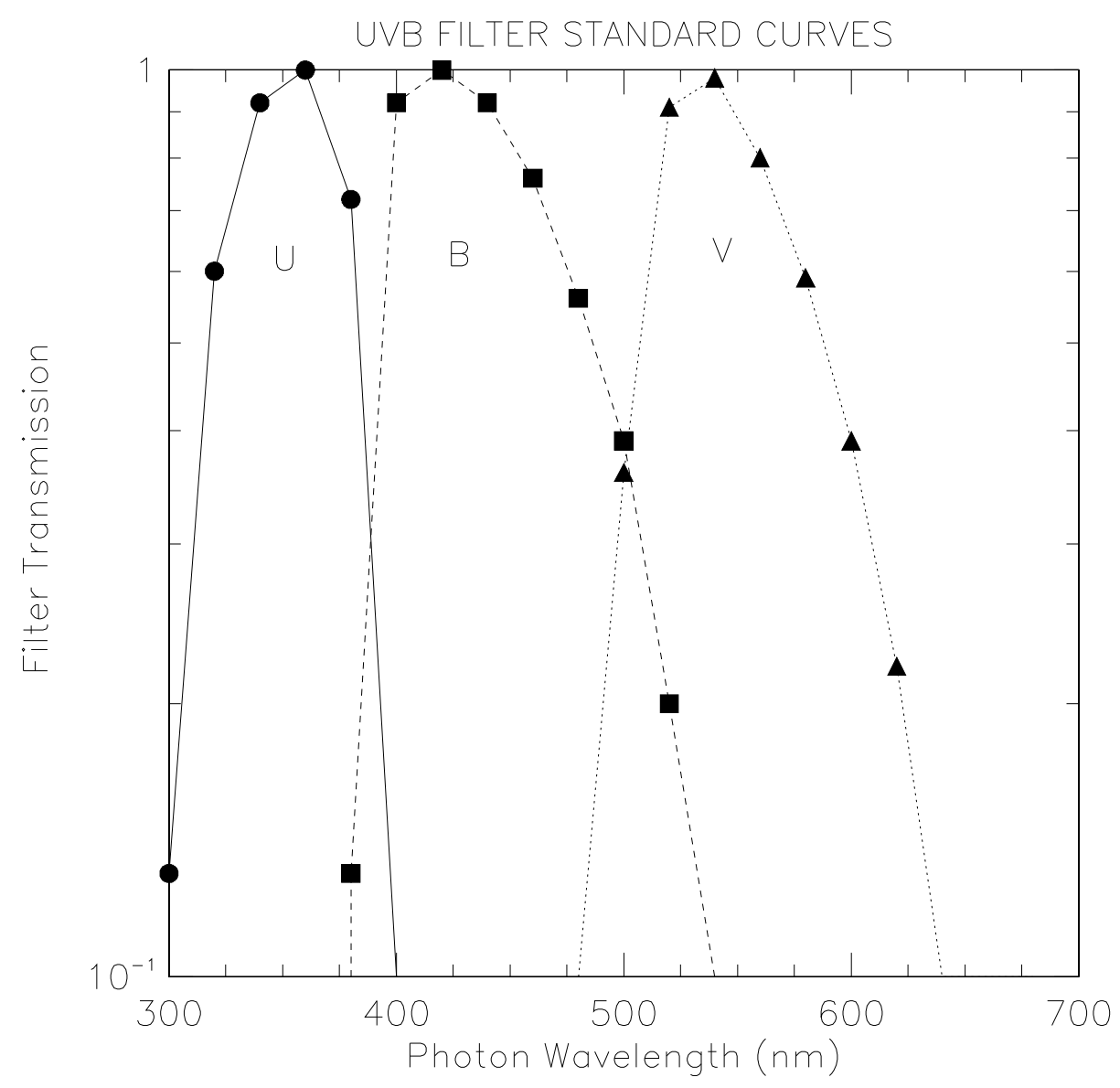

Figure 3.3: Response curves for the standard photometric UBV filters 20. 


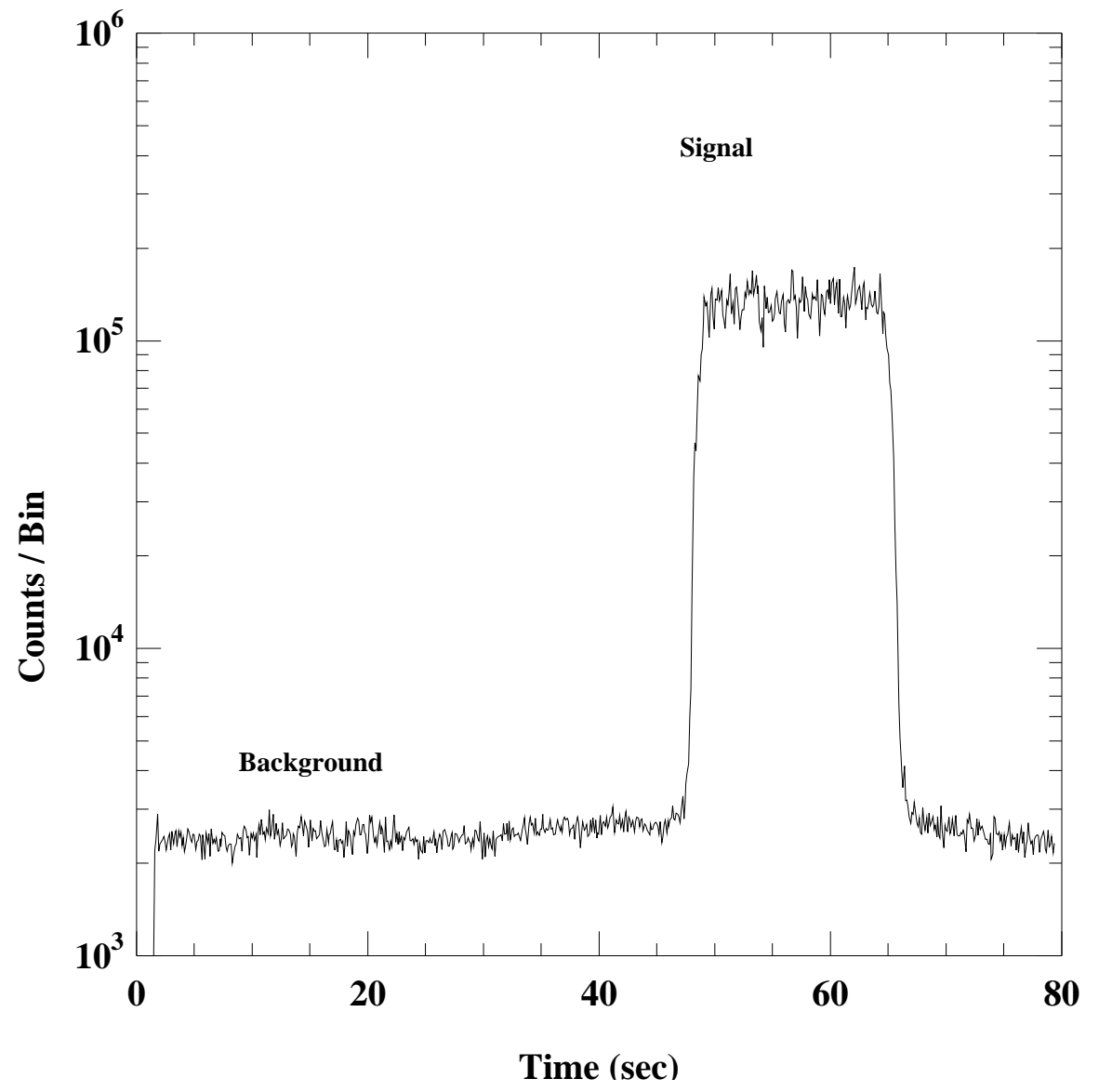

Figure 3.4: Light curve for the star Mu Andromeda showing the PMT counting rate for a single drift scan using the V-band filter. Time bins are 0.1sec. 


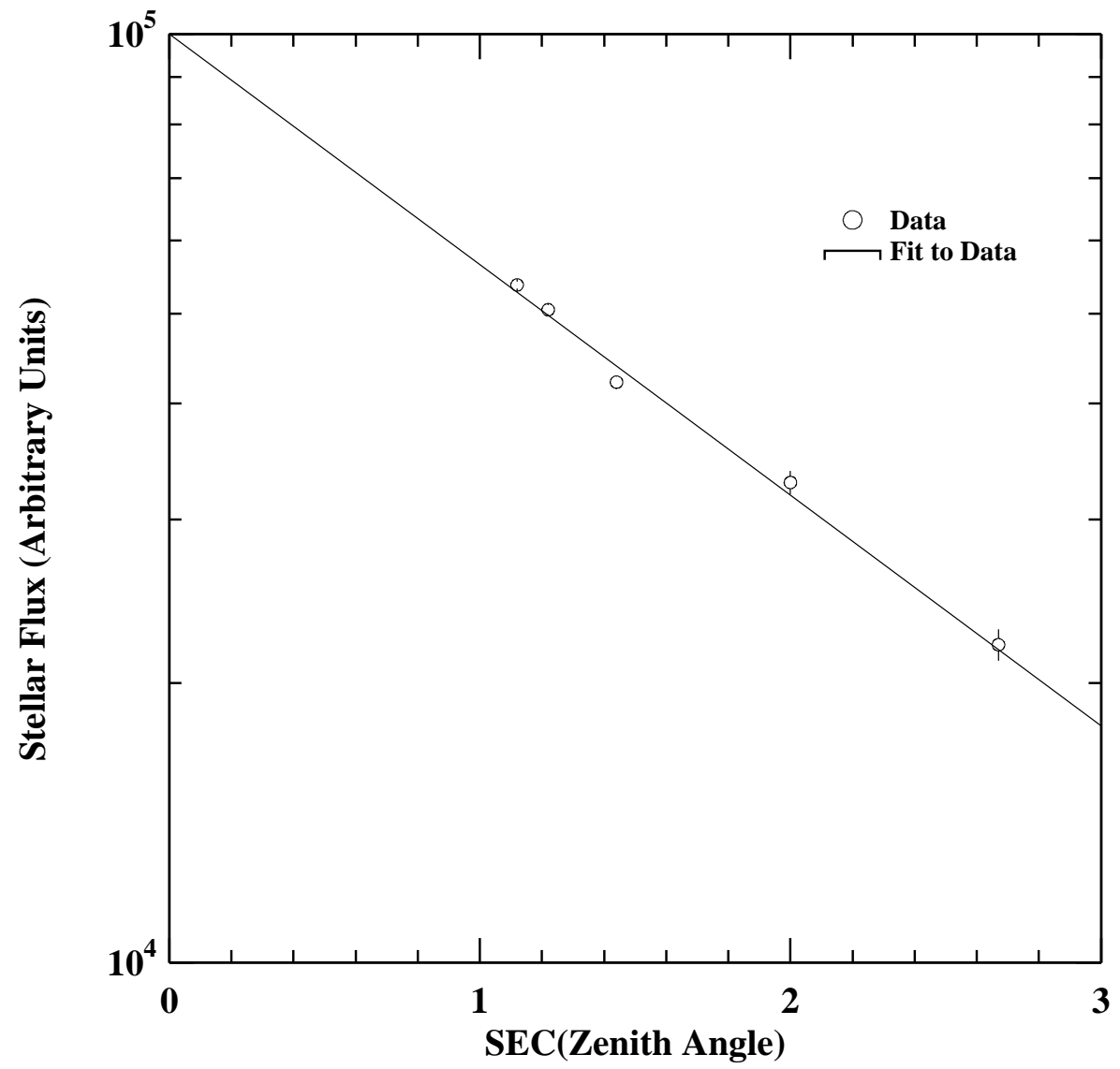

Figure 3.5: Plot of stellar flux versus atmospheric depth for the star Mu Andromeda. 


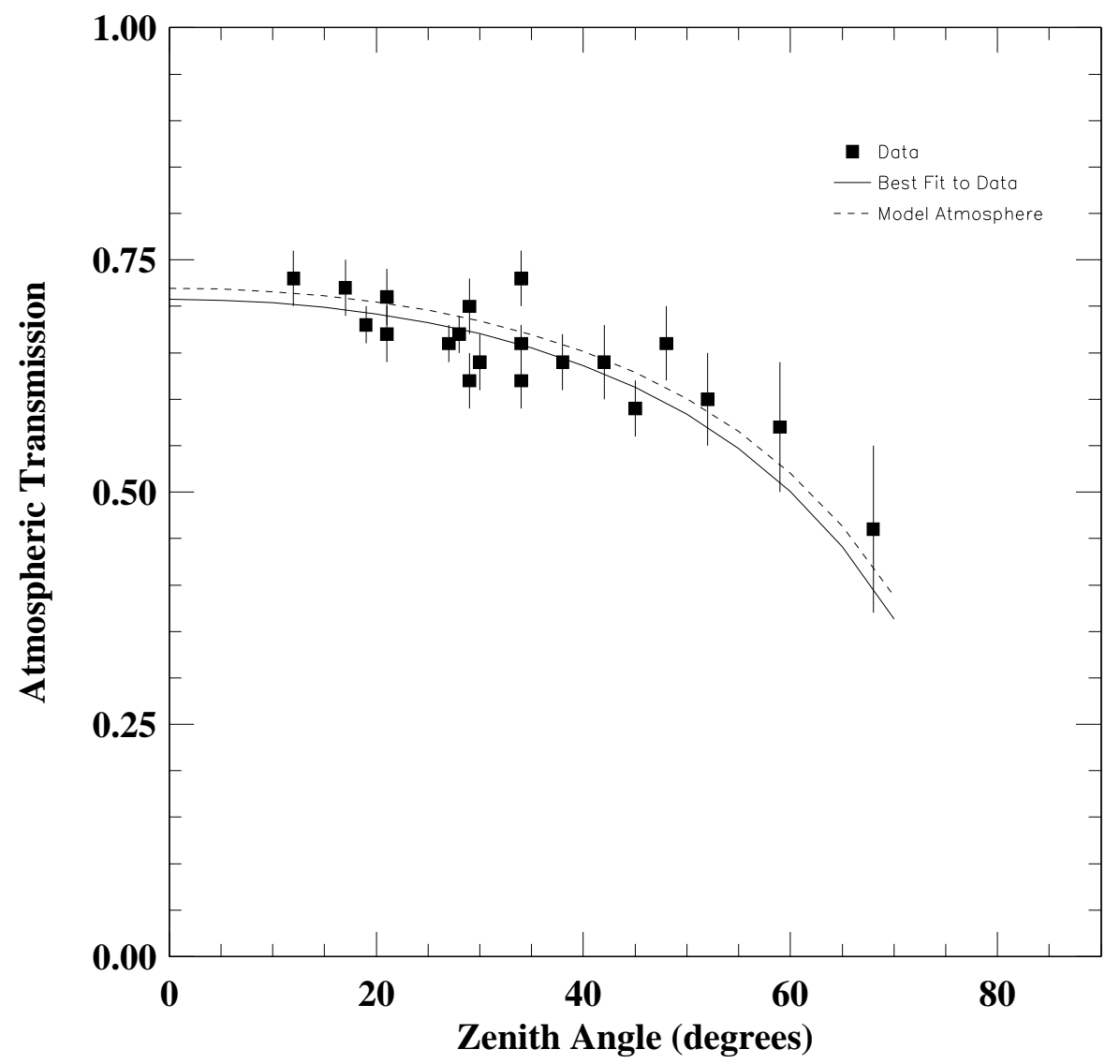

Figure 3.6: Atmospheric transmission for the blue waveband, which contains most of the observable Cherenkov light, as a function of zenith angle. The points and curves are identified in the legend. 


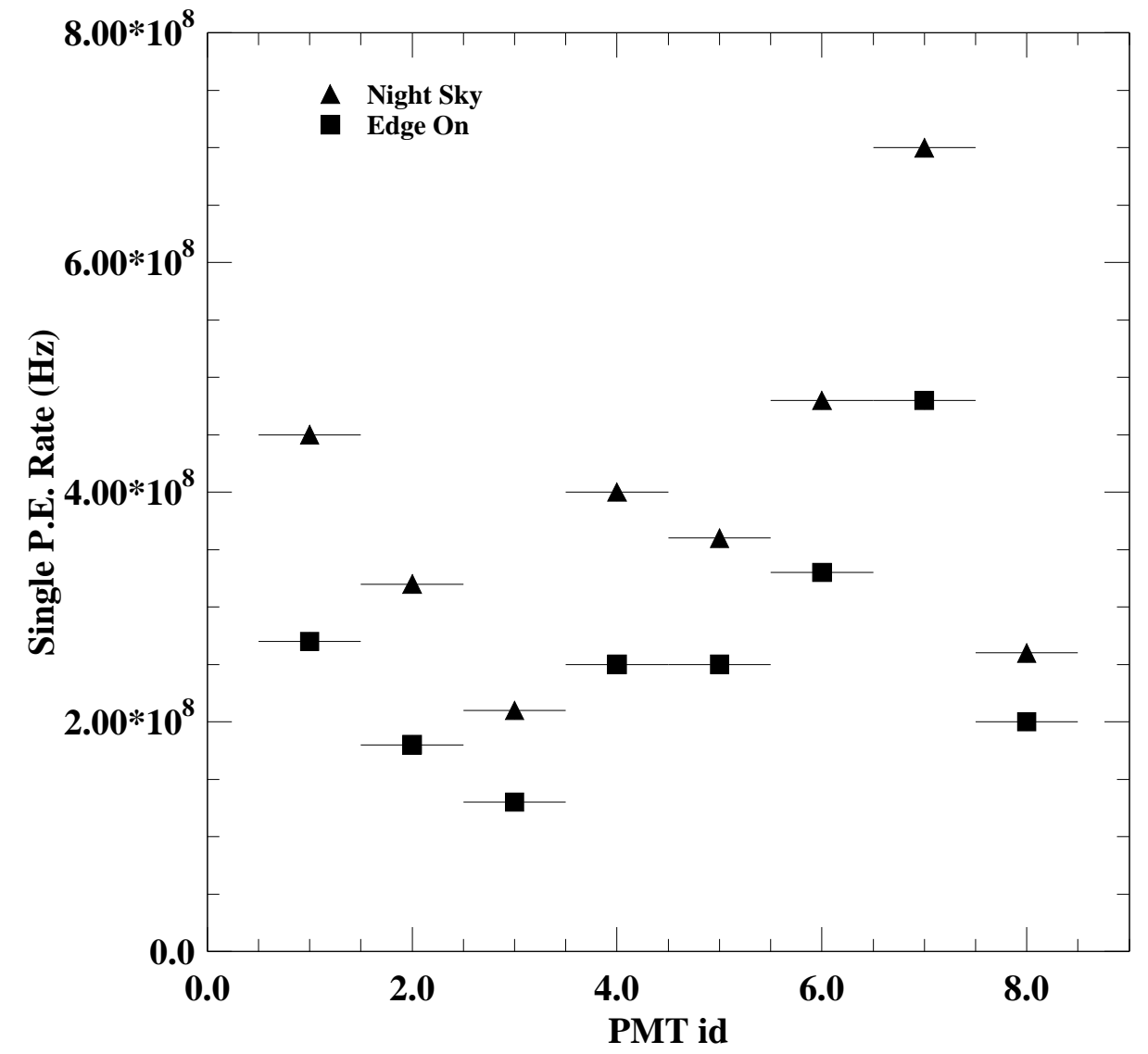

Figure 3.7: Single photoelectron rates for the camera PMTs for two heliostat conditions: edge facing the secondary, and viewing the night sky. Note that the statistical errors on the data are smaller than the point sizes. 

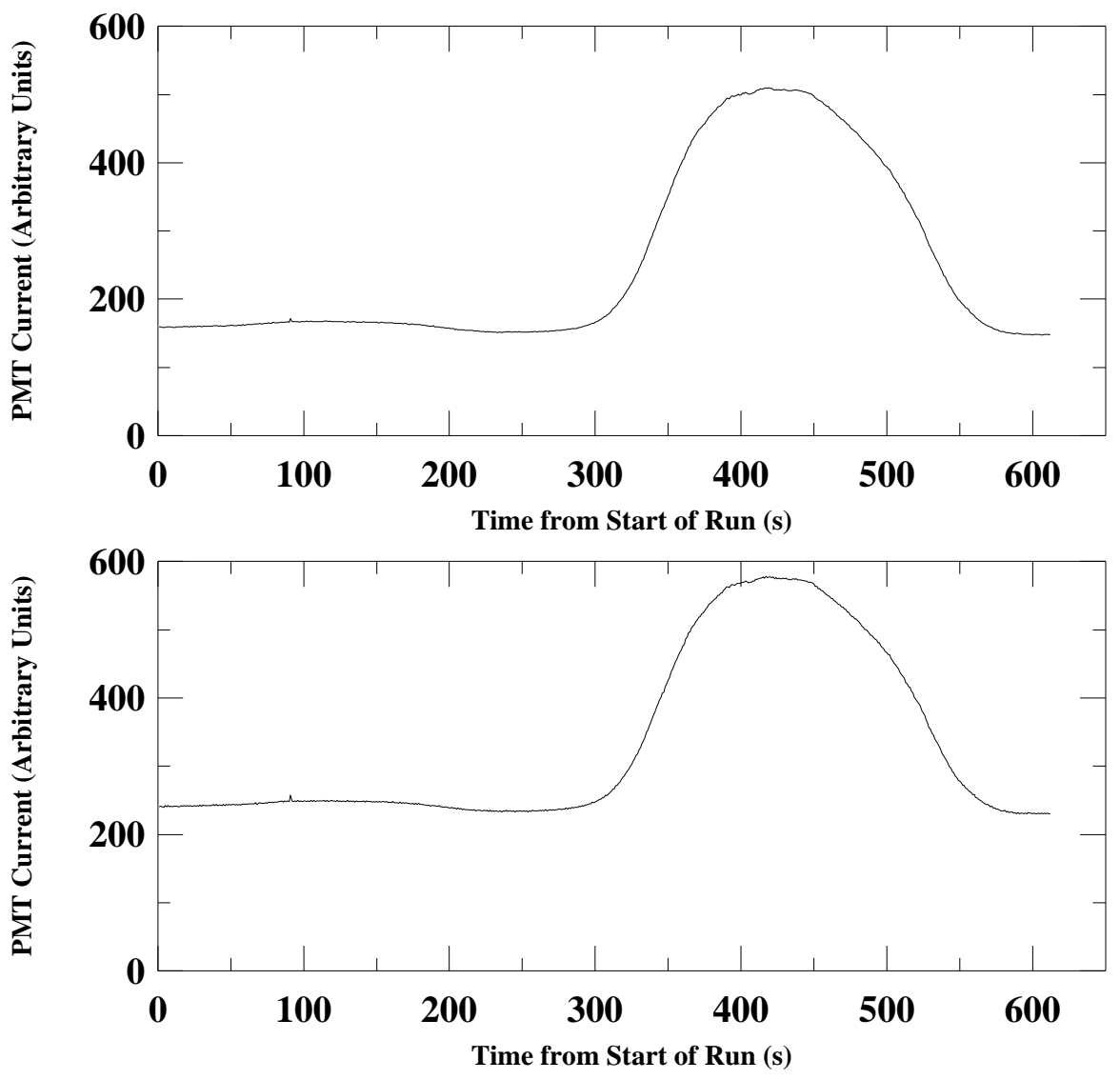

Figure 4.1: Currents in two PMTs during a drift scan of the star Aldebaran. 

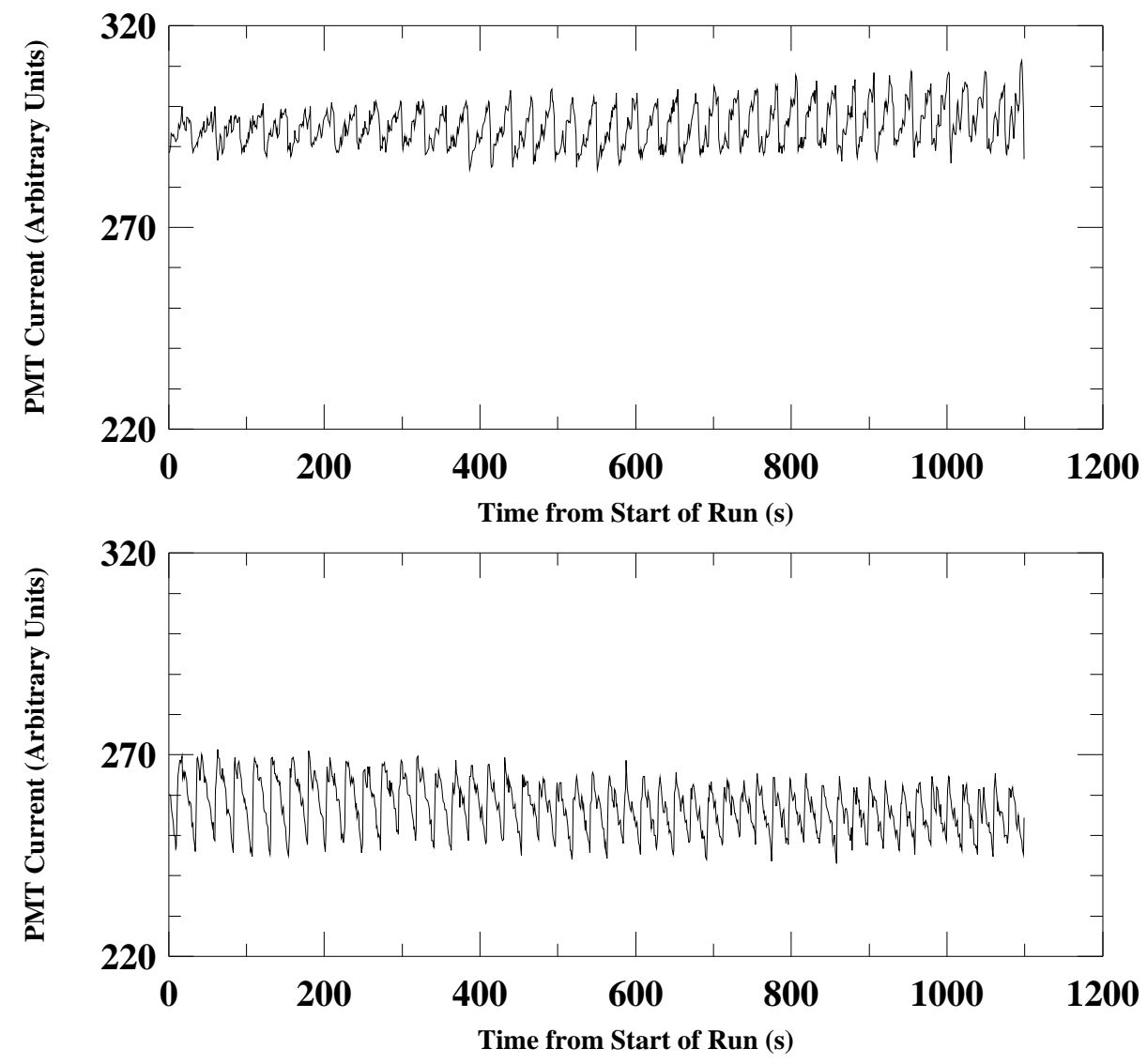

Figure 4.2: PMT currents for two channels recorded while the heliostats were tracking a bright star. Note the suppressed zeroes on the vertical scales. 


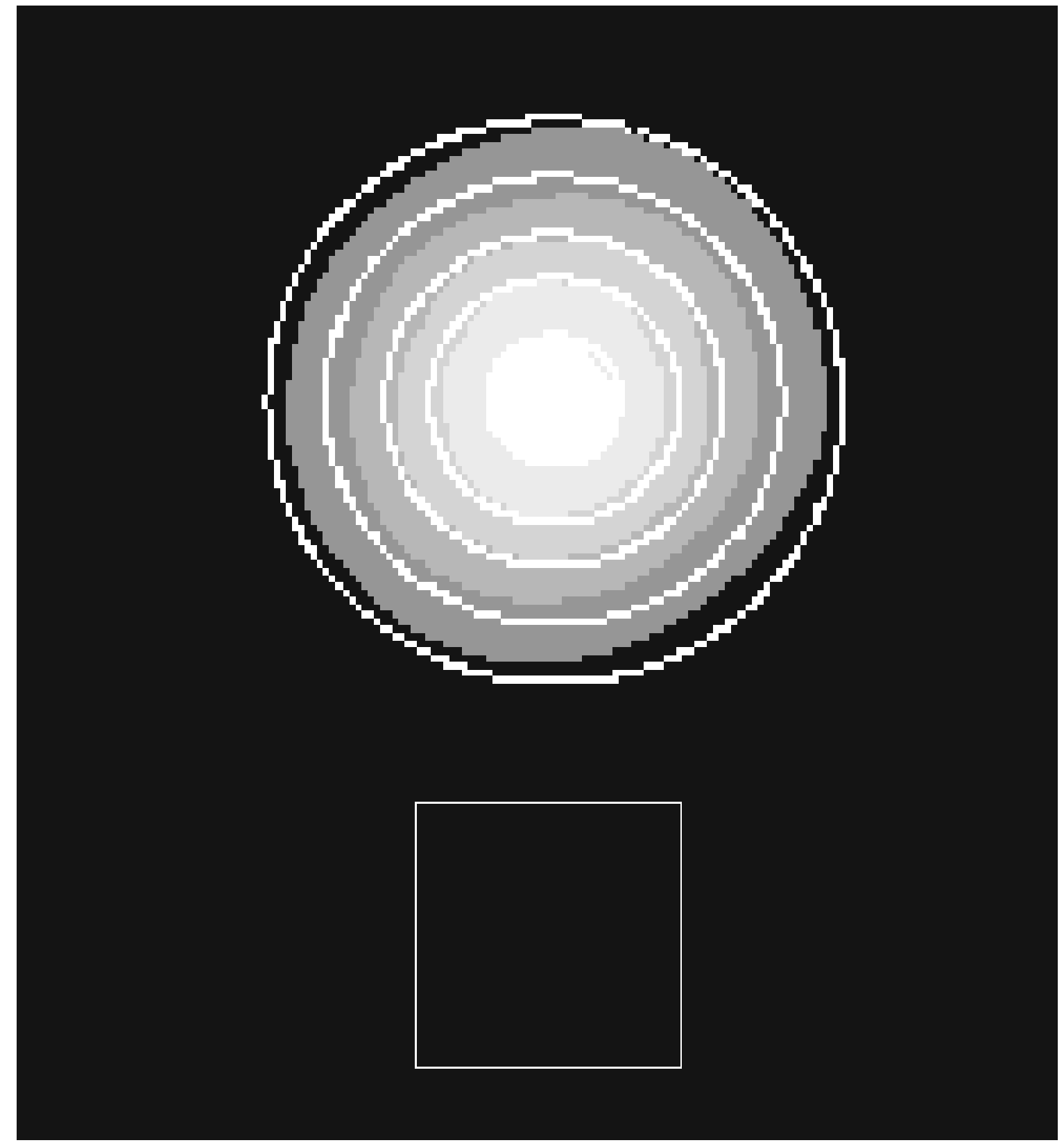

Figure 4.3: CCD image of a single heliostat Sun spot projected onto the tower. The contours represent the fraction of the total light contained within a given radius, starting at $10 \%$ and increasing in steps of $20 \%$. The box underneath is a $1 m \times 1 m$ box to indicate scale. 


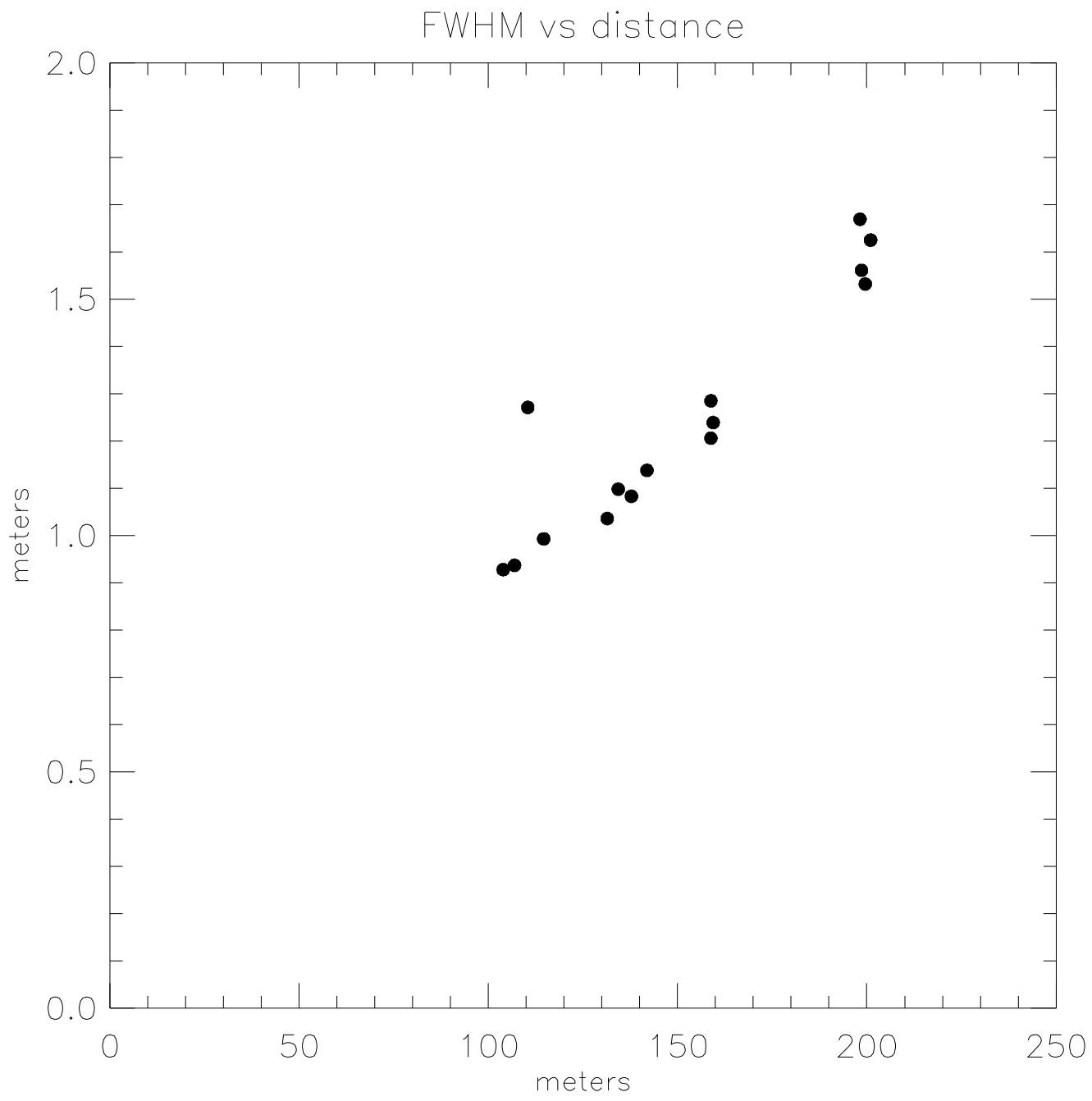

Figure 4.4: Solar spot size (FWHM) projected on a target at the tower versus distance between heliostat and target for 15 heliostats measured using a CCD camera. Except for one heliostat with particularly poor optical alignment $(8 \mathrm{E} 3$ at $107 \mathrm{~m})$ the spots follow a regular trend, becoming less concentrated with greater distance from the tower. 


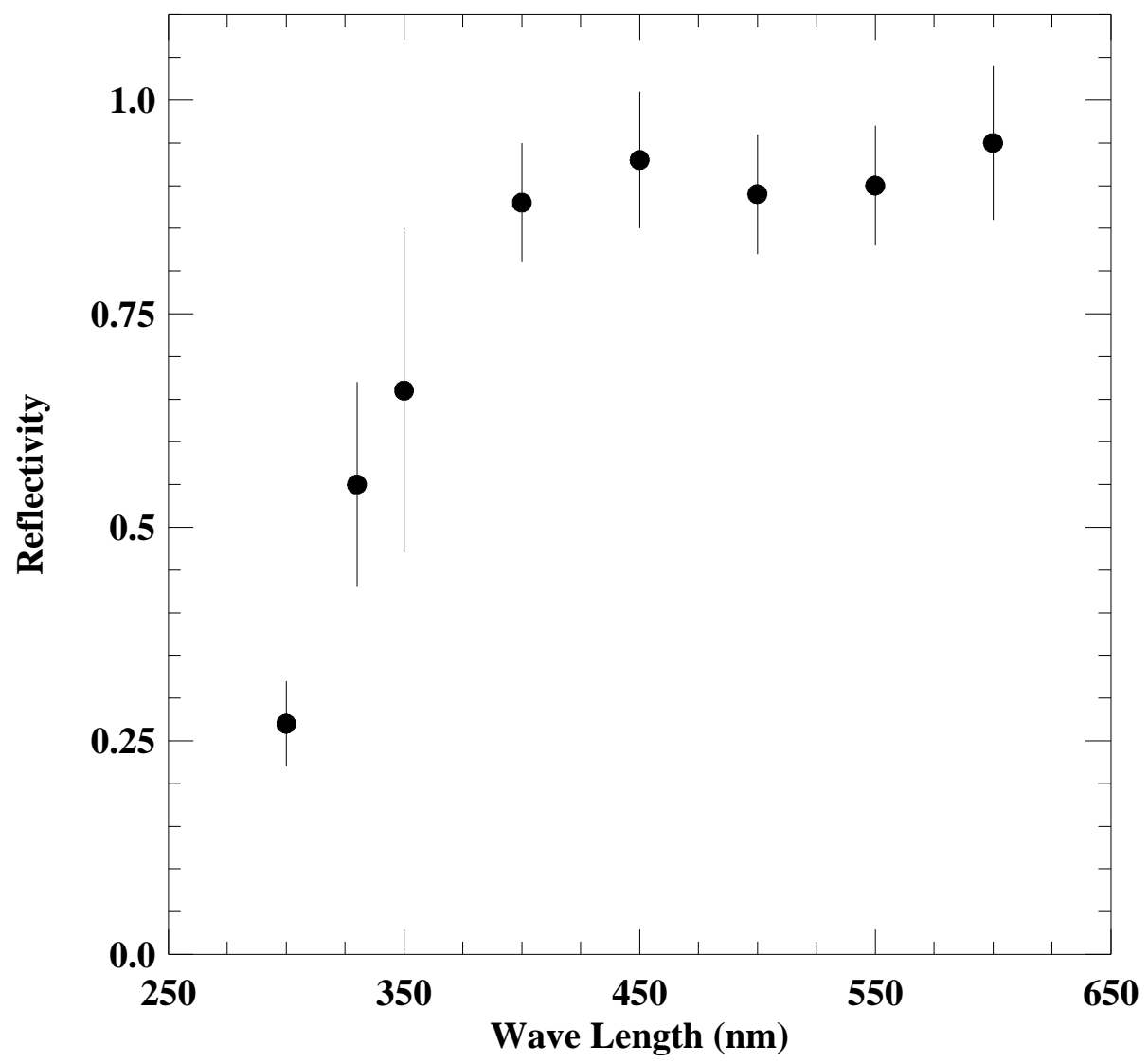

Figure 4.5: Measured heliostat facet reflectivity, as a function of wavelength. 


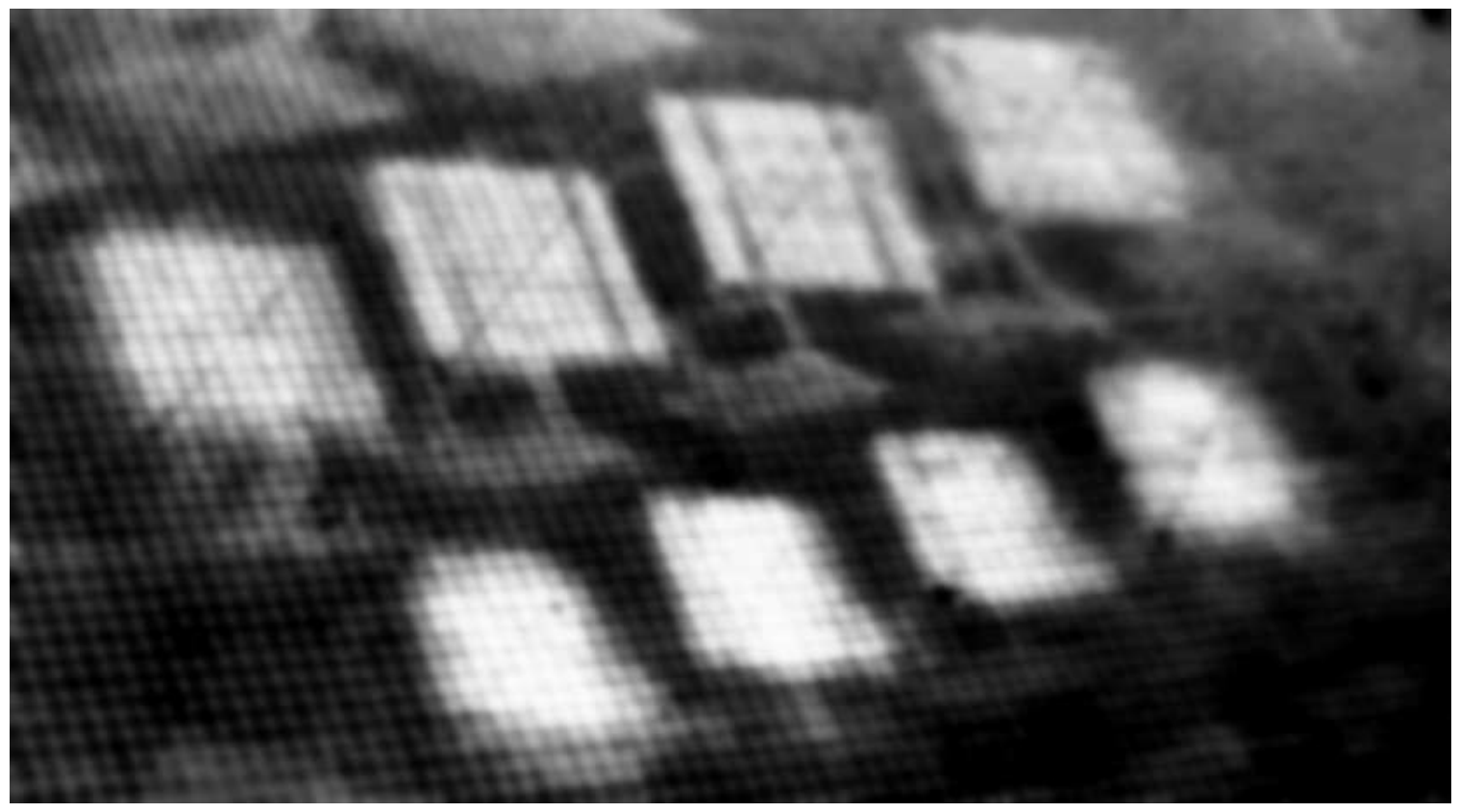

Figure 5.1: A CCD image of the Sandia heliostat field projected onto a white lucite placard at the focal plane of the secondary used for the October 1996 observations. This image was obtained by viewing the placard at an oblique angle from the balcony where the telescope mount is installed. Grid lines drawn on the placard represent a $1 \mathrm{~cm}$ spacing at the focal plane. Images of eight selected heliostats are distinct and well-separated, with virtually no optical overlap. 


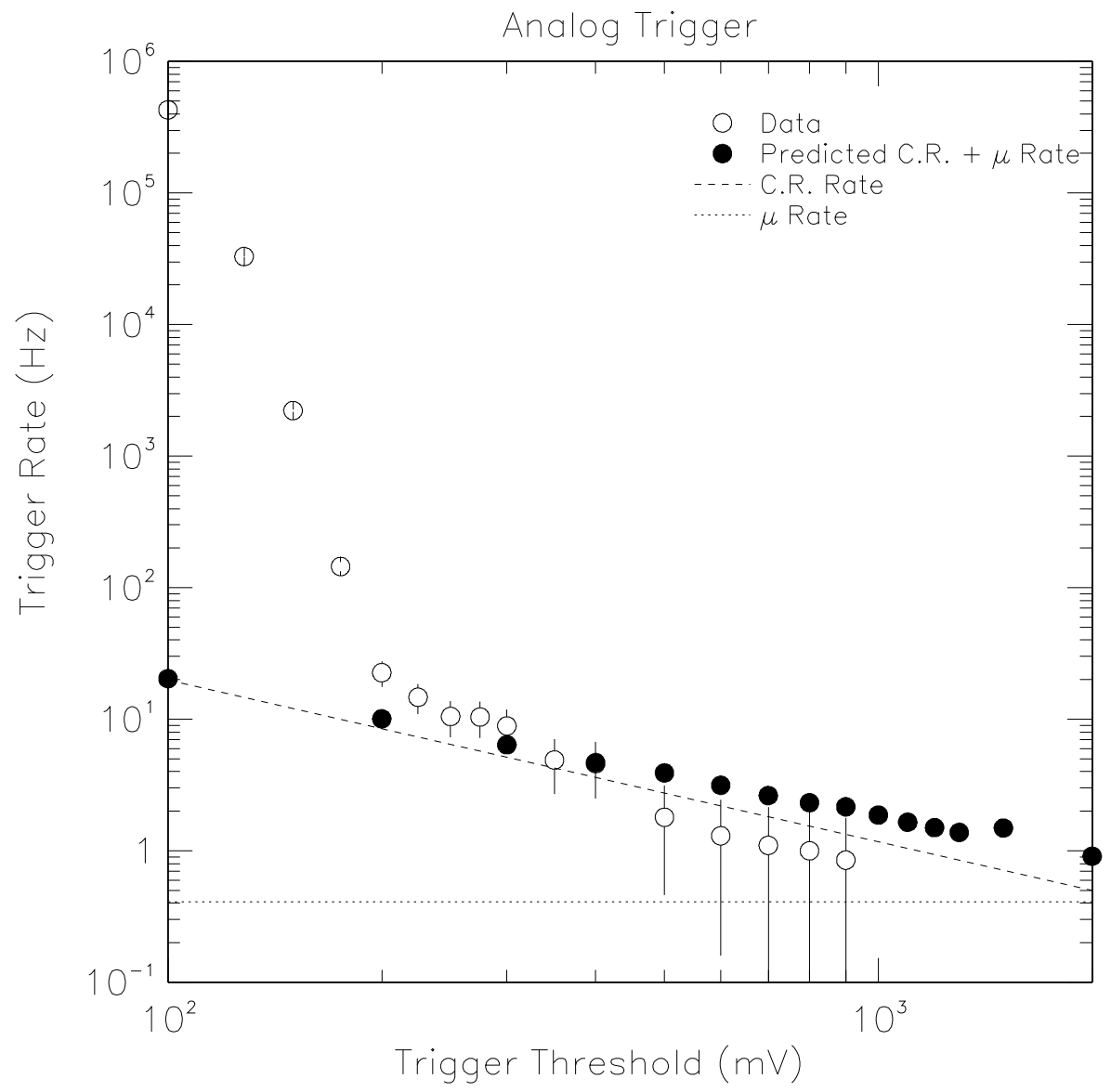

Figure 5.2: Analog trigger rate as a function of discriminator threshold. The data are compared to a model of single heliostat triggers from cosmic rays and muons, as identified in the legend. The heliostats were viewing separate parts of the sky. 


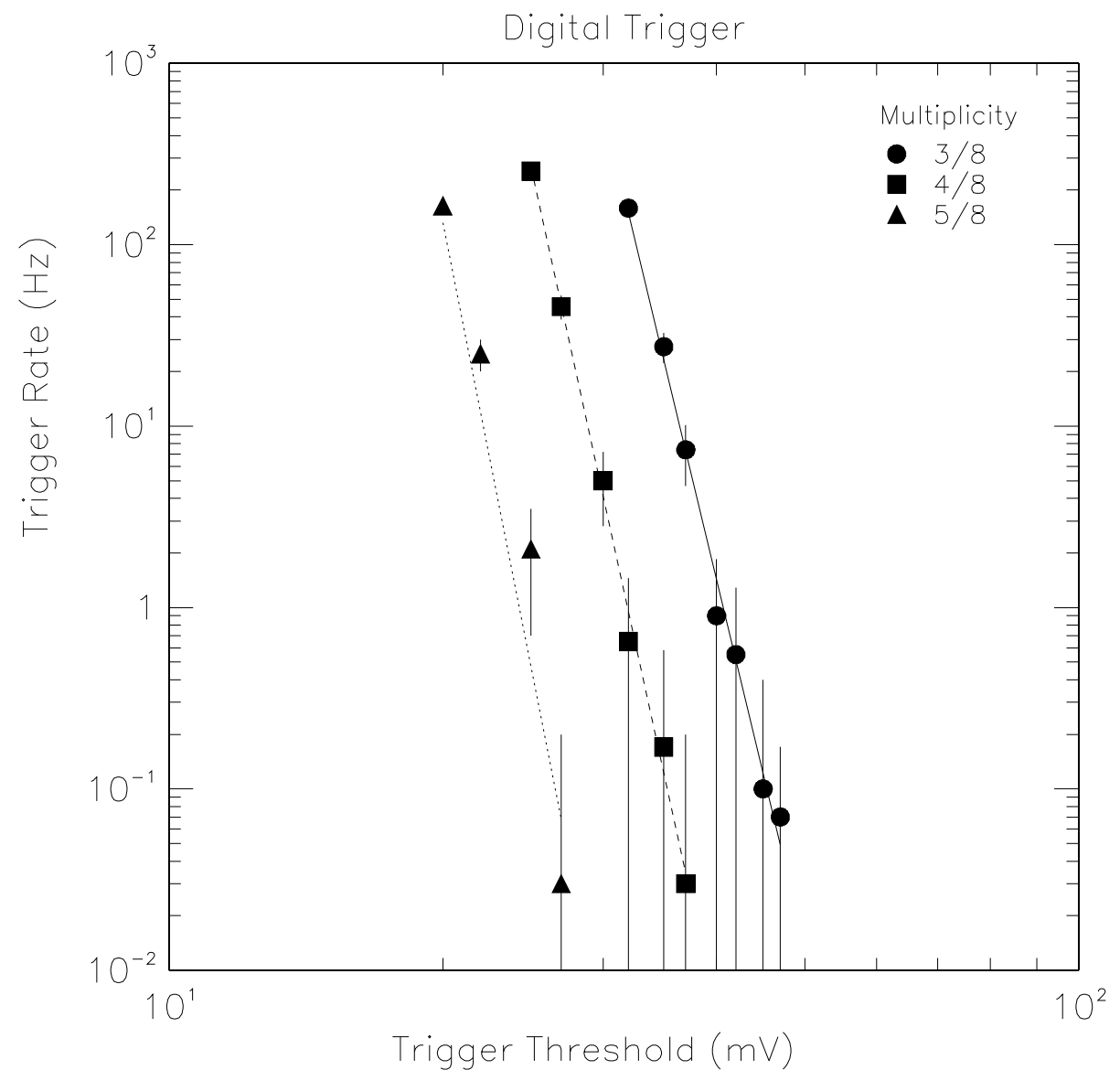

Figure 5.3: Digital trigger rate as a function of individual channel discriminator threshold. The heliostats were viewing separate parts of the sky. The lines represent power law fits to the data, for different trigger configurations, as identified in the legend. 


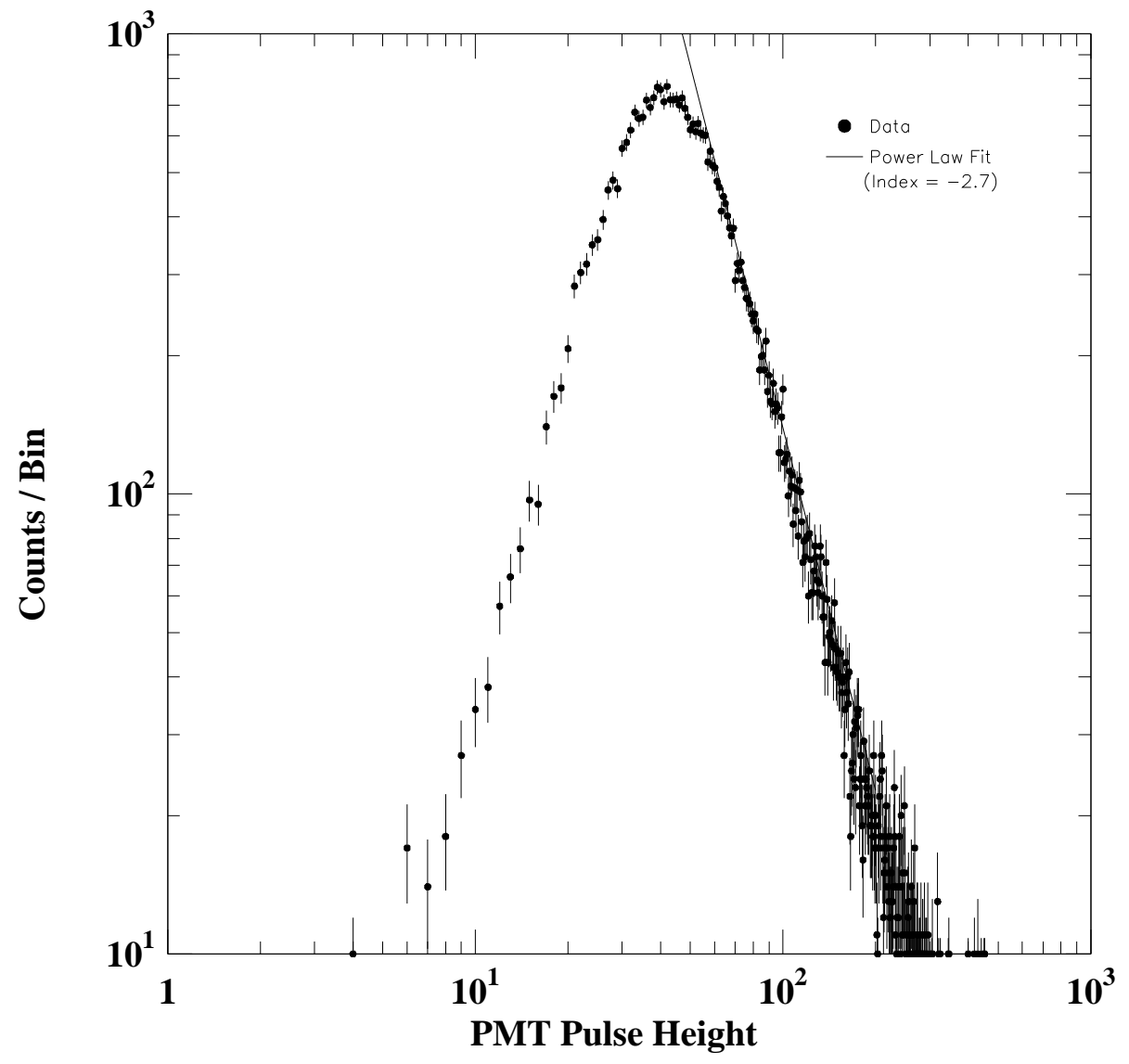

Figure 5.4: Pulse height spectrum obtained from observations of air showers at the zenith. 


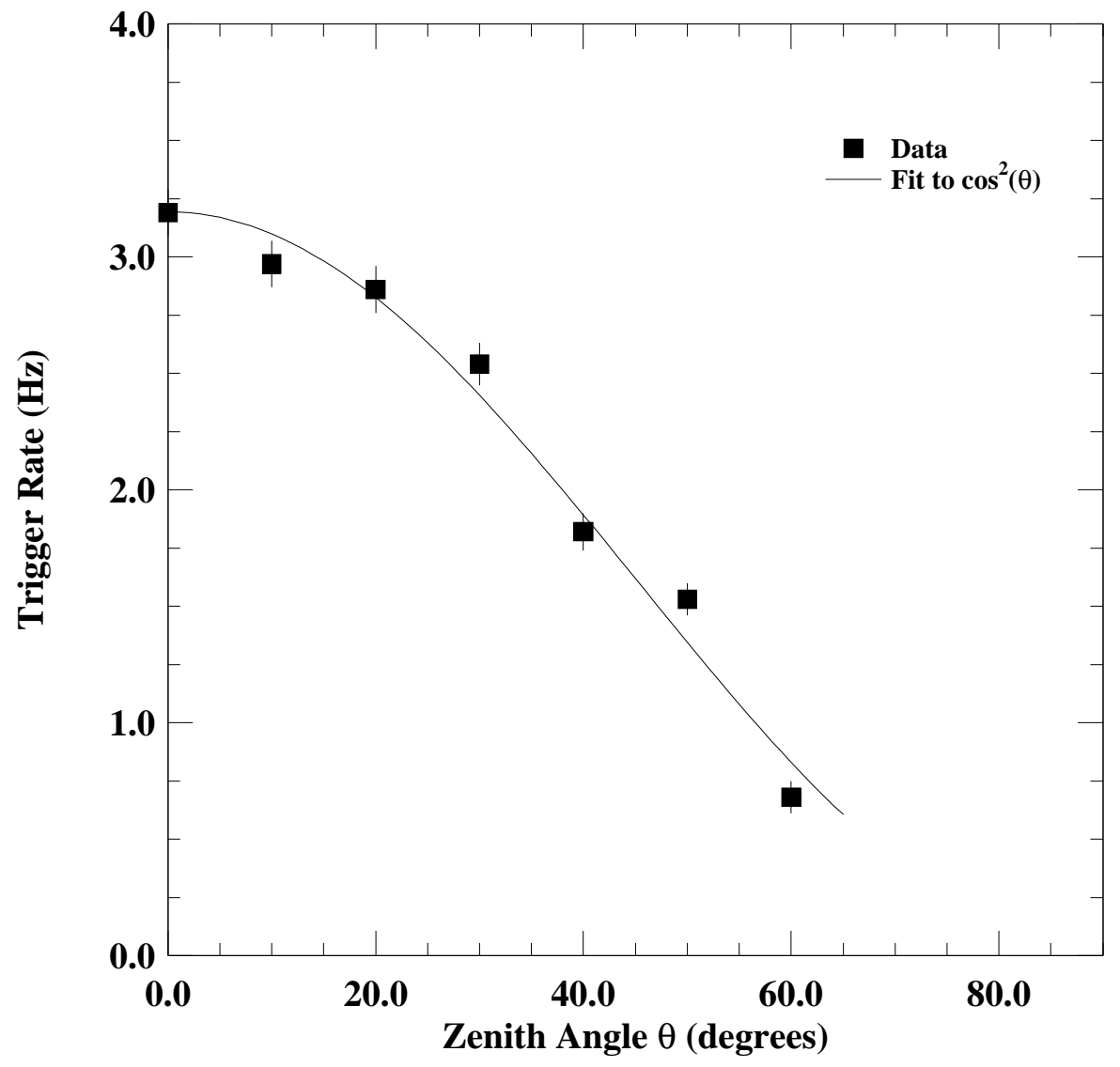

Figure 5.5: Trigger rate as a function of zenith angle. 


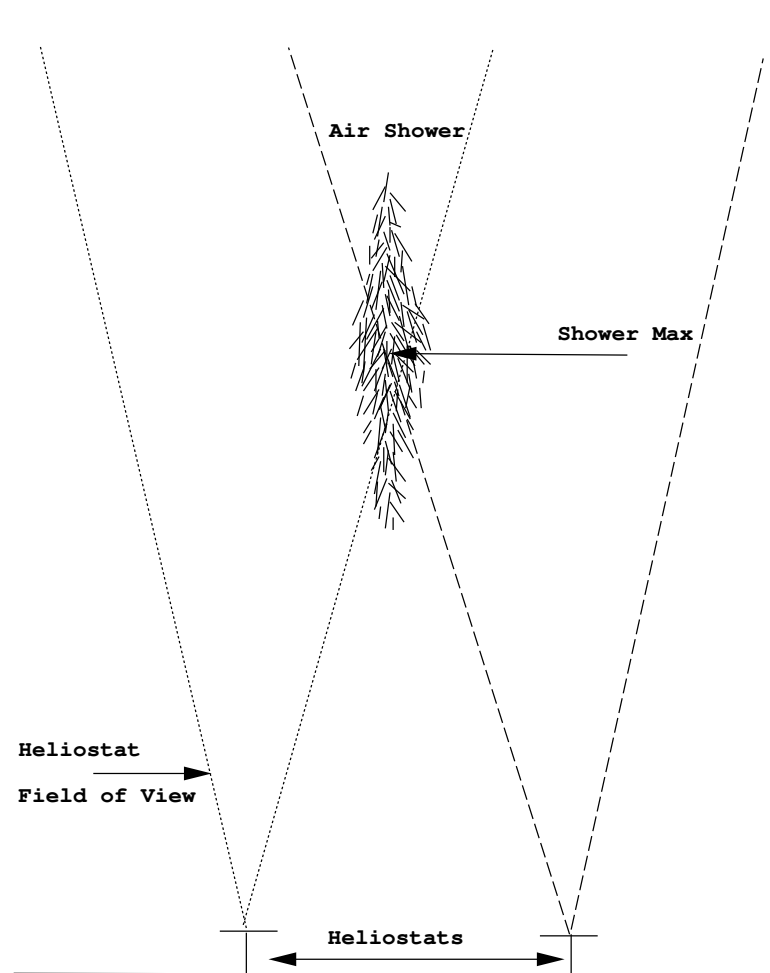

Figure 5.6a: Diagram illustrating overlapping heliostat fields-of-view when the heliostats are all aimed at a source at infinity. Note that the entire track of the air shower is not completely contained within the overlap of the heliostat fields-of-view. Angles and distances have been exaggerated for purposes of illustrating the concept.

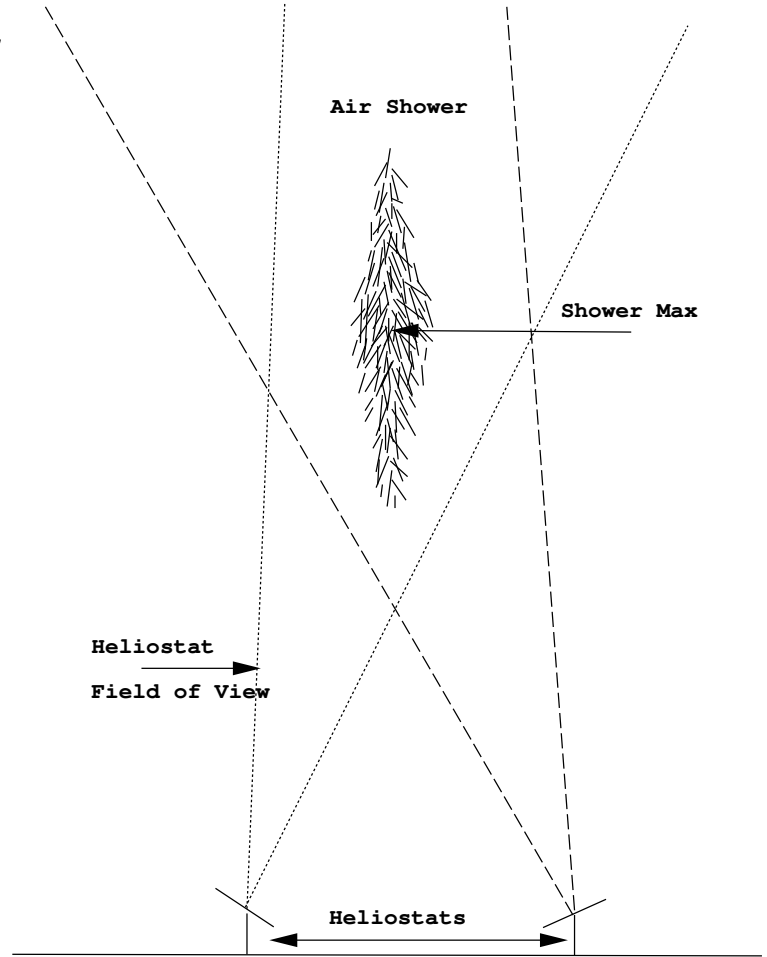

Figure 5.6b: Diagram illustrating the advantage of convergent viewing. When the heliostats are canted in to view the interaction region the air shower is better contained by the overlapping heliostat fields-of-view. 


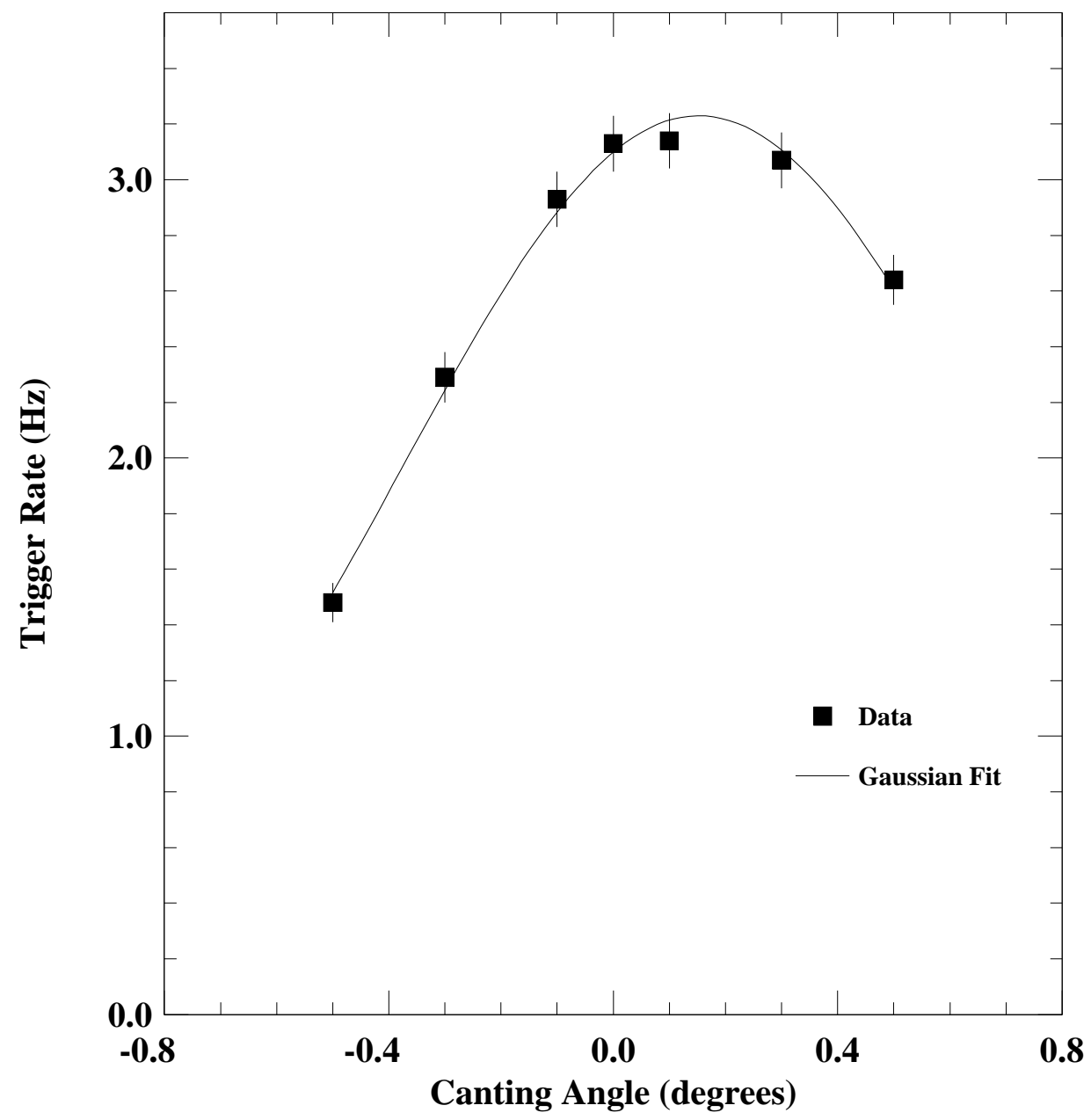

Figure 5.7: Trigger rates as a function of heliostat canting angle. A canting angle of $0^{\circ}$ corresponds to parallel viewing. A 4/8 digital trigger was used and the heliostats were viewing the zenith. 


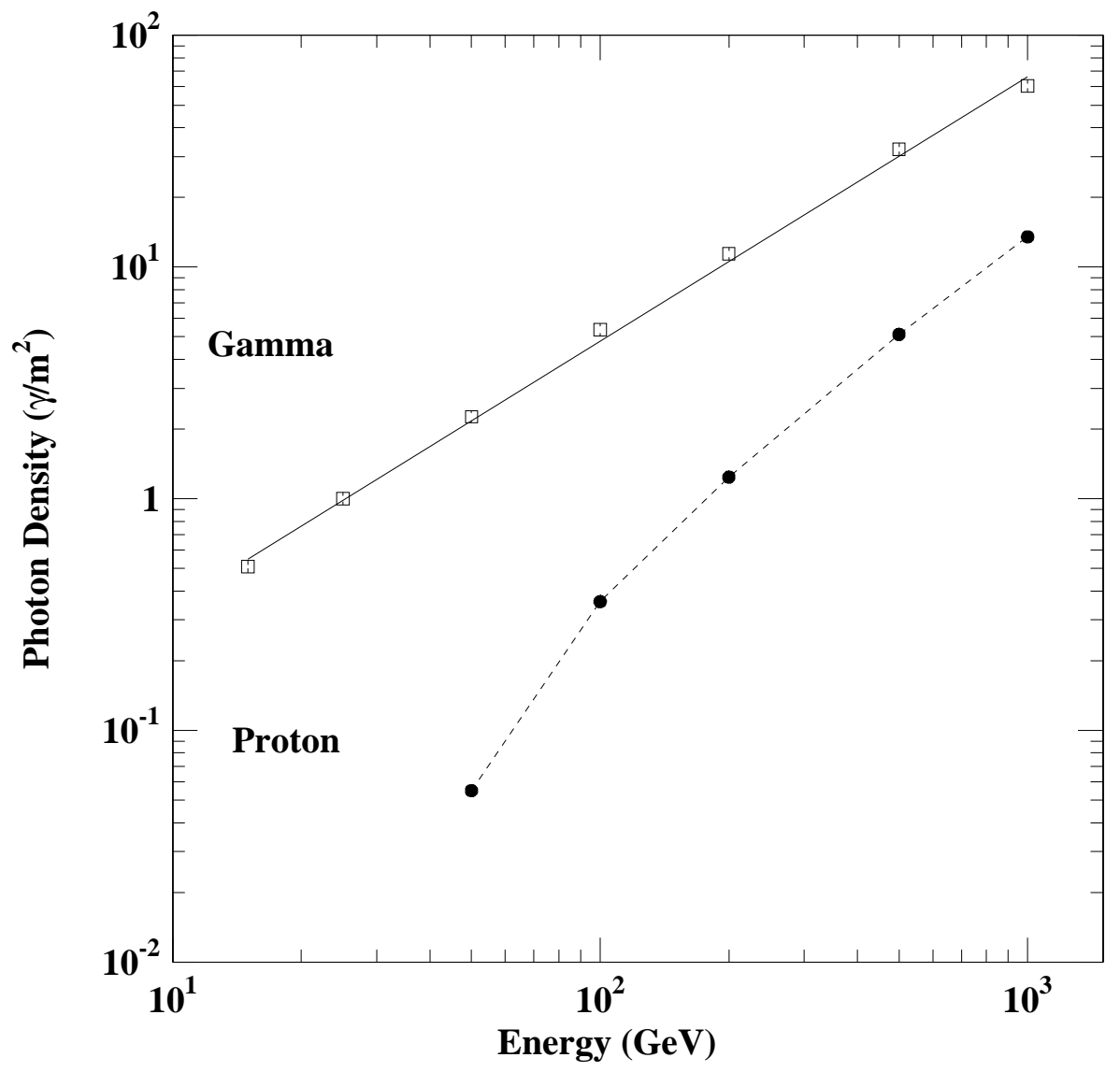

Figure 5.8: The density of Cherenkov photons on the ground, within $100 \mathrm{~m}$ of the core, for vertically incident $\gamma$-ray and proton initiated air showers as determined by the Hillas Monte Carlo. The measured field of view of the Sandia heliostats has been accounted for so that only those photons which would be collected by the STACEE secondary telescope have been considered. Note that the statistical errors are smaller that the size of the symbols used in plotting the data. 